\title{
Direct One Pot Synthesis of Phenanthrenes via Suzuki-Miyaura Coupling/Aldol Condensation Cascade Reaction
}

Young Ha Kim,${ }^{\dagger}$ Hyuk Lee, Yeong Joon Kim,${ }^{\dagger}$ Bum Tae Kim, and Jung-Nyoung Heo*

The Center for Medicinal Chemistry, Korea Research Institute of Chemical Technology, 100 Jang-dong, Daejeon 305-600, Korea, 'Department of Chemistry, Chungnam National University, 220 Gung-dong, Daejeon 305-764, Korea

E-mail: heojn@krict.re.kr

\section{SUPPORTING INFORMATION}

Experimental details and spectroscopic data for compounds $\mathbf{1 b}, \mathbf{2 b}, \mathbf{3}, \mathbf{4 a - q}, \mathbf{8 a - d , 1 0 ,}$ and 11. This material is available free of charge via the Internet at http://pubs.acs.org 


\section{General}

All reactions were conducted in oven-dried microwave glassware by using Biotage Initiator $\mathrm{EXP}^{\mathrm{TM}}$ microwave reactor under an atmosphere of dry nitrogen. All solvents were purified before use unless otherwise indicated. Toluene was distilled from $\mathrm{CaH}_{2}$. All other reagents were used without further purification.

Aryl bromides, $\mathbf{1 a}^{1}$ and $\mathbf{1 c}{ }^{2}$ were prepared by the coupling of 2-bromophenylacetic acid with methylamine and benzylamine, respectively. $1 \mathbf{d}^{3}$ and $\mathbf{1} \mathbf{k}^{4}$ were prepared by following the literature methods. Phenylacetates ${ }^{5}$ 1e and $\mathbf{1 f}$ were prepared by reaction of 2-bromophenylacetic acid in the presence of catalytic $p$-toluenesulfonic acid, in methanol and in ethanol, respectively. Compounds $\mathbf{1 g - 1 \mathbf { j }}, \mathbf{1 1}, \mathbf{2 a}, \mathbf{2 c}, \mathbf{7}$, and $\mathbf{6 a - d}$ were obtained from commercial sources and used without further purification.

Analytical thin layer chromatography (TLC) was performed on Kieselgel 60 $\mathrm{F}_{254}$ glass plates precoated with a $0.2 \mathrm{~mm}$ thickness of silica gel. The TLC plates were visualized by shortwave $(254 \mathrm{~nm})$ or longwave $(360 \mathrm{~nm})$ UV light, potassium permanganate or ceric ammonium molybdate stain. Flash chromatography on Kieselgel 60 (230400 mesh) silica gel was performed using a CombiFlash Companion system. Preparative HPLC normal phase separations were performed using a Shimadzu HPLC system composed of two LC-8A pumps, a CTO-10A column oven and injector, a SPD10A detector, and a SCL-10A system controller.

\footnotetext{
1 Sielecki, T. M.; Meyers, A. I. J. Org. Chem. 1992, 57, 3673-3676.

${ }^{2}$ Wolfe, J. P.; Rennels, R. A.; Buchwald, S. L. Tetrahedron 1996, 52, 7525-7546.

${ }^{3}$ Lebegue, N.; Bethegnies, G.; Berthelot, P. Synth. Commun. 2004, 34, 1041-1048.

${ }^{4}$ Baker, R. W.; Foulkes, M. A.; Griggs, M.; Nguyen, B. N. Tetrahedron Lett. 2002, 43, 9319-9322.

5 Bashore, C. G.; Samardjiev, I. J.; Bordner, J.; Coe, J. W. J. Am. Chem. Soc. 2003, 125, 3268-3272.
} 
FT-IR spectra were recorded as neat samples using a Travel IR Portable spectrometer. Melting points were determined on a Uni-Melt capillary melting point apparatus and are uncorrected. GC/MS spectra were measured on a Shimadzu spectrometer. ${ }^{1} \mathrm{H}$ NMR and spectra were obtained at $300 \mathrm{MHz}$ on a Varian Gemini 300 instrument using $\mathrm{CDCl}_{3}$ as solvent. ${ }^{1} \mathrm{H}$ NMR assignment abbreviations are the following: singlet (s), doublet (d), triplet (t), quartet (q), broad singlet (bs), doublet of doublets (dd), doublet of triplets (dt), and multiplet (m). ${ }^{13} \mathrm{C}$ NMR spectra were measured at $75.5 \mathrm{MHz}$ or $125 \mathrm{MHz}$ using $\mathrm{CDCl}_{3}$ as an internal reference.<smiles>CNC(=O)Cc1ccc(OC)cc1Br</smiles>

2-(2-Bromo-4-methoxyphenyl)- $N$-methylacetamide (1b). To a solution of (2-bromo4-methoxy)phenylacetic acid (1.0 g, $4.08 \mathrm{mmol})$ in $10 \mathrm{~mL}$ of $\mathrm{CH}_{2} \mathrm{Cl}_{2}$ was added methylamine $\left(0.23 \mathrm{~mL}, 40 \mathrm{wt} \%\right.$ in $\left.\mathrm{H}_{2} \mathrm{O}, 2.7 \mathrm{mmol}\right)$ and $\mathrm{EDCI}(0.78 \mathrm{~g}, 4.08 \mathrm{mmol})$. The mixture was stirred at room temperature for $2 \mathrm{~h}$ and quenched with $\mathrm{H}_{2} \mathrm{O}(10 \mathrm{~mL})$. The resulting two phases were separated and the aqueous layer was extracted with $\mathrm{CH}_{2} \mathrm{Cl}_{2}(2$ $\times 10 \mathrm{~mL}$ ). The combined organic layers washed with brine and dried over $\mathrm{MgSO}_{4}$, and concentrated in vacuo. The residue was purified by flash column chromatography (50\% $\rightarrow 80 \%$ EtOAc/hexanes) to provide acetamide $1 \mathbf{b}(683 \mathrm{mg})$ in $65 \%$ yield as a white solid: mp 98 100 ; ${ }^{1} \mathrm{H}$ NMR (300 MHz, $\left.\mathrm{CDCl}_{3}\right) \delta 7.24(\mathrm{~d}, 1 \mathrm{H}, J=8.5 \mathrm{~Hz}), 7.14(\mathrm{~d}$, $1 \mathrm{H}, J=2.6 \mathrm{~Hz}), 6.86(\mathrm{dd}, 1 \mathrm{H}, J=8.5,2.6 \mathrm{~Hz}), 5.53(\mathrm{bs}, 1 \mathrm{H}), 3.79(\mathrm{~s}, 1 \mathrm{H}), 3.64(\mathrm{~s}, 1 \mathrm{H})$, $2.77(\mathrm{~d}, 3 \mathrm{H}, J=4.8 \mathrm{~Hz}) ;{ }^{13} \mathrm{C} \mathrm{NMR}\left(75 \mathrm{MHz}, \mathrm{CDCl}_{3}\right) \delta 170.9,159.7,132.3,127.0$, 
125.4, 118.6, 114.3, 55.8, 43.2, 26.7; IR (neat) 3288, 1650, 1496, 1255, $1029 \mathrm{~cm}^{-1}$; MS (EI) $\mathrm{m} / z \mathrm{M}^{+}$for $\mathrm{C}_{10} \mathrm{H}_{12} \mathrm{BrNO}_{2}$ calcd 258, found $258\left(\mathrm{M}^{+}, 2\right), 200$ (83), 198 (78), 178 (100), 150 (10), $121(20)$.<smiles>COc1cc(Br)c(C(=O)c2cc(OC)c(OC)cc2OC)cc1OC</smiles>

2b

4,5-Dimethoxy-2-formylphenylboronic acid (2b). The literature procedure ${ }^{6}$ was used to prepare the title compound (51\% yield for two steps), which was obtained as a white solid: mp 200 202 ; $\quad{ }^{1} \mathrm{H} \mathrm{NMR}\left(300 \mathrm{MHz}, \mathrm{CDCl}_{3}\right) \delta 9.78(\mathrm{~s}, 1 \mathrm{H}), 8.03(\mathrm{~s}, 2 \mathrm{H}), 7.82$ (s, 1H), $7.38(\mathrm{~s}, 1 \mathrm{H}), 4.05(\mathrm{~s}, 3 \mathrm{H}), 4.01(\mathrm{~s}, 3 \mathrm{H}) ;{ }^{13} \mathrm{C} \mathrm{NMR}\left(75 \mathrm{MHz}, \mathrm{CDCl}_{3}\right) \delta 196.8,153.4$, 150.5, 133.7, 120.9, 120.8, 56.5, 56.3; MS (EI) $m / z \mathrm{M}^{+}$for $\mathrm{C}_{9} \mathrm{H}_{11} \mathrm{BO}_{5}$ calcd 210, found $210\left(\mathrm{M}^{+}, 58\right), 209(29), 179$ (29), 166 (100).

${ }^{6}$ Geen, G. R.; Mann, I. S.; Mullane, M. V. Tetrahedron 1998, 54, 9875-9894. 


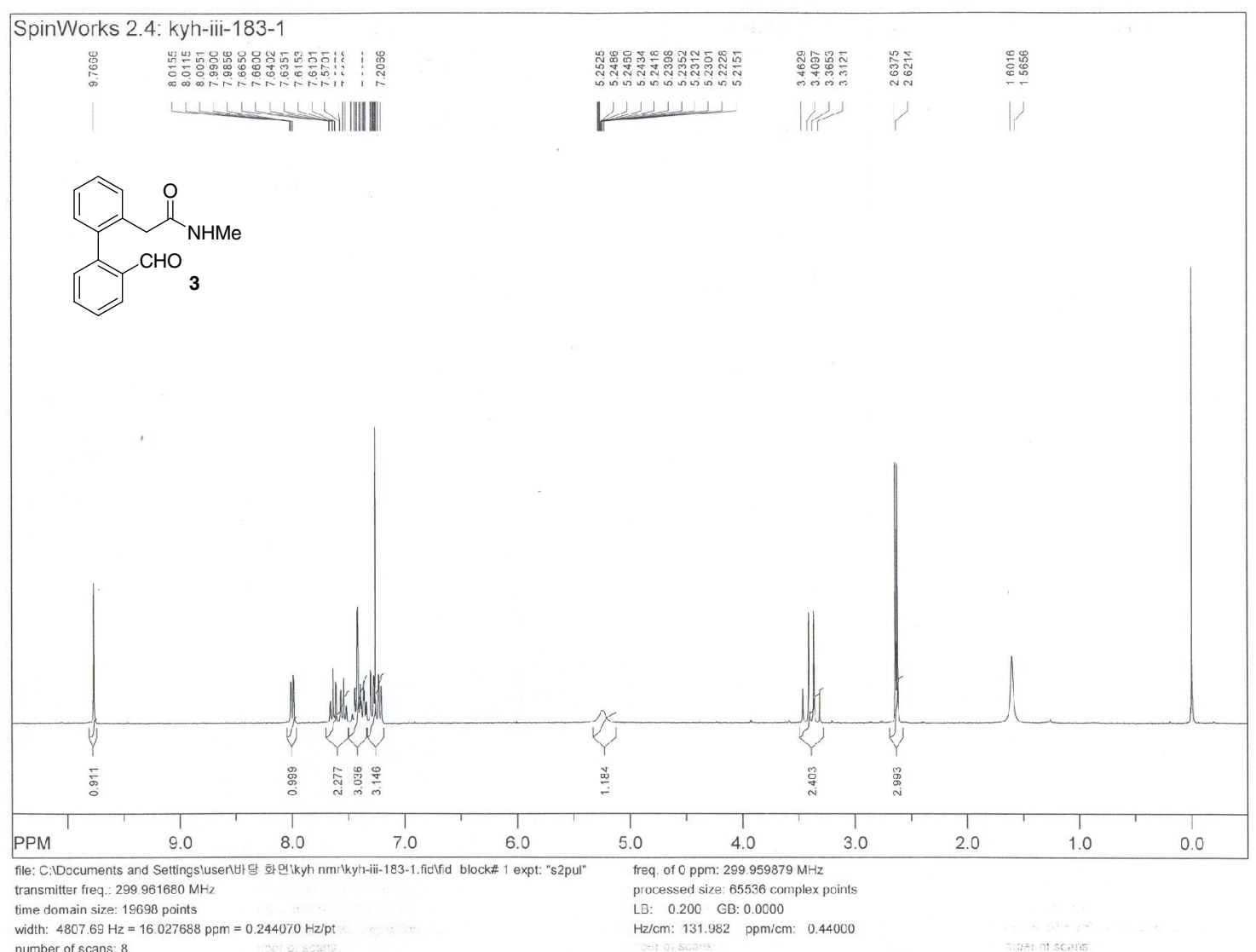

number of scans: 8

$2=5.10120095$

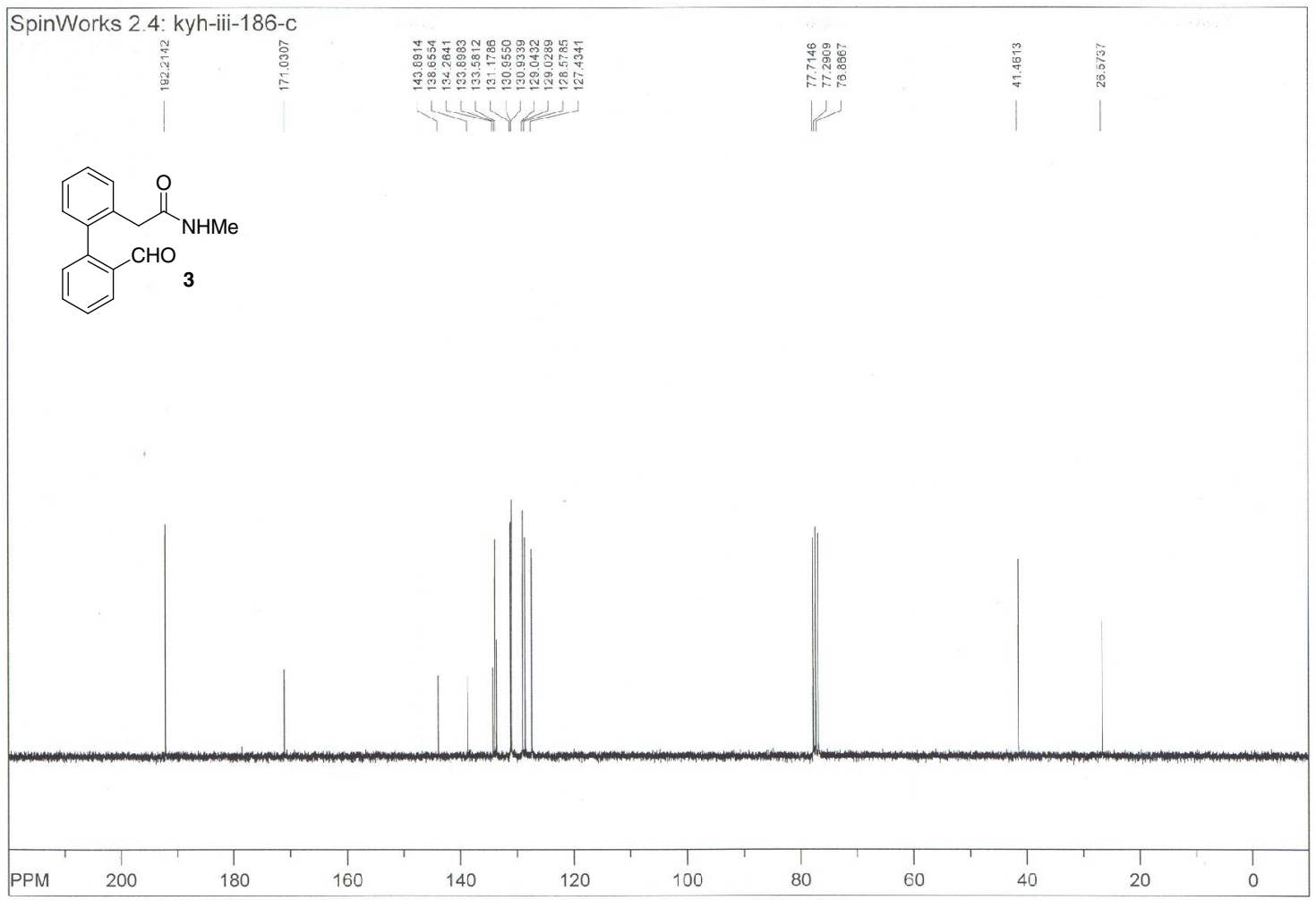

file: C:IDocuments and SettingsiKRICT비 탕 화면 $k y$ h-ii-186c.filfid block\# 1 expt: "s2pul" freq. of 0 ppm: $75.424954 \mathrm{MHz}$

transmitter freq.: $75.432873 \mathrm{MHz}$ processed size: 65536 complex points

time domain size: 47120 points

processed size: 65536 complex points
LB: 0.000 GB: 0.0000

width: $18115.94 \mathrm{~Hz}=240.159776 \mathrm{ppm}=0.384464 \mathrm{~Hz} / \mathrm{pt}$

Hz/cm: $693.910 \mathrm{ppm} / \mathrm{cm}: 9.19903$

number of scans 51 ? 


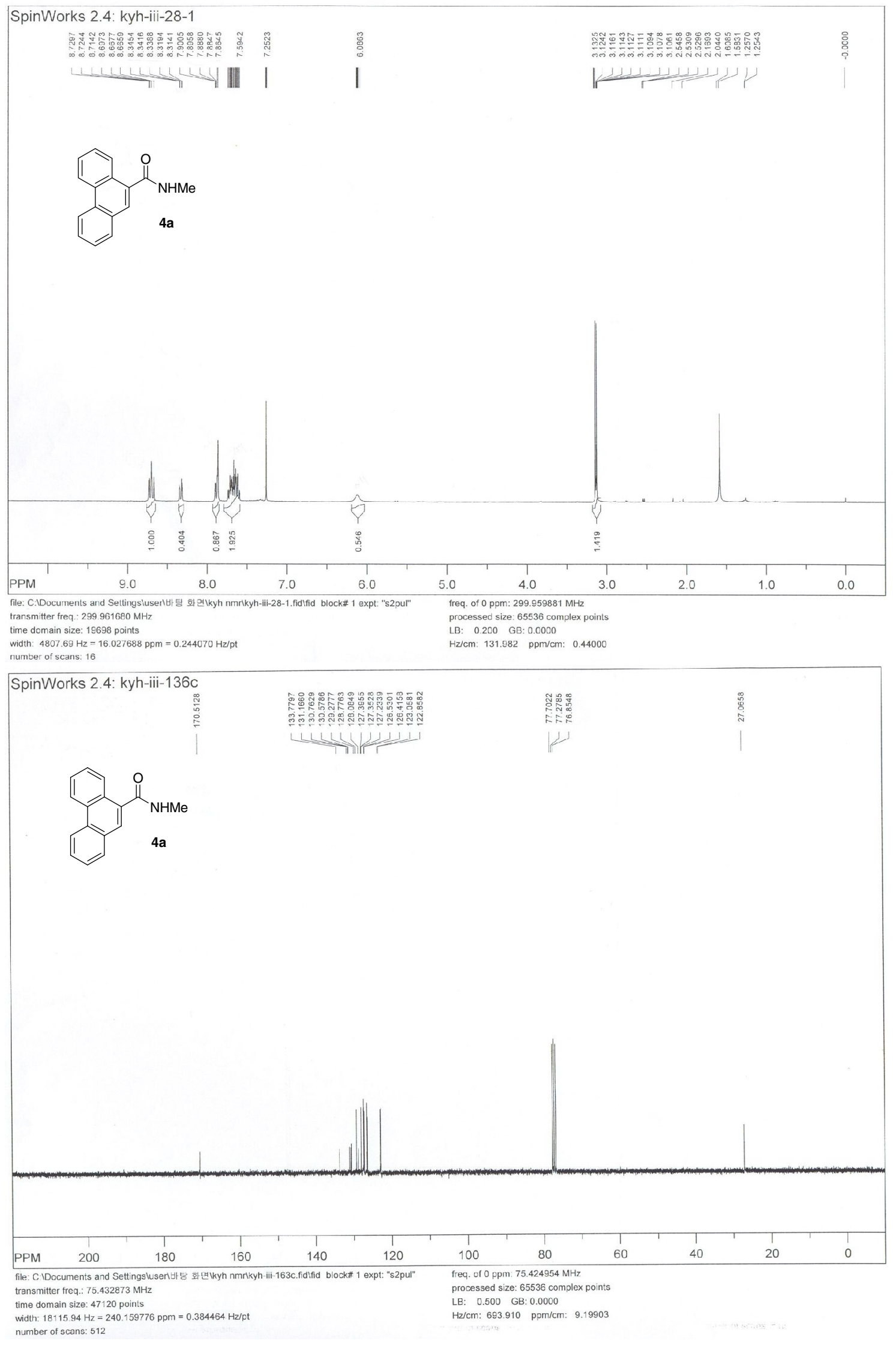




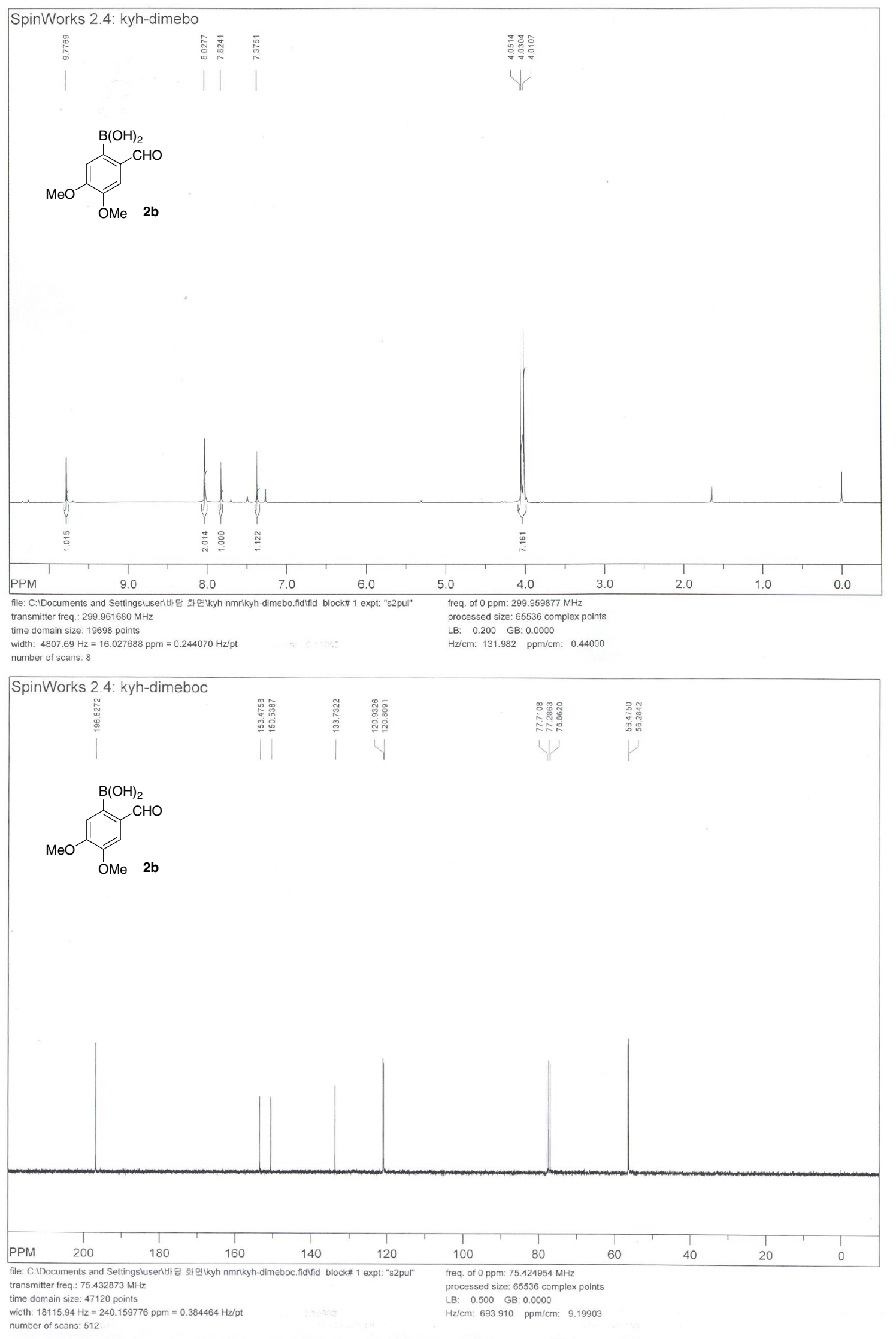




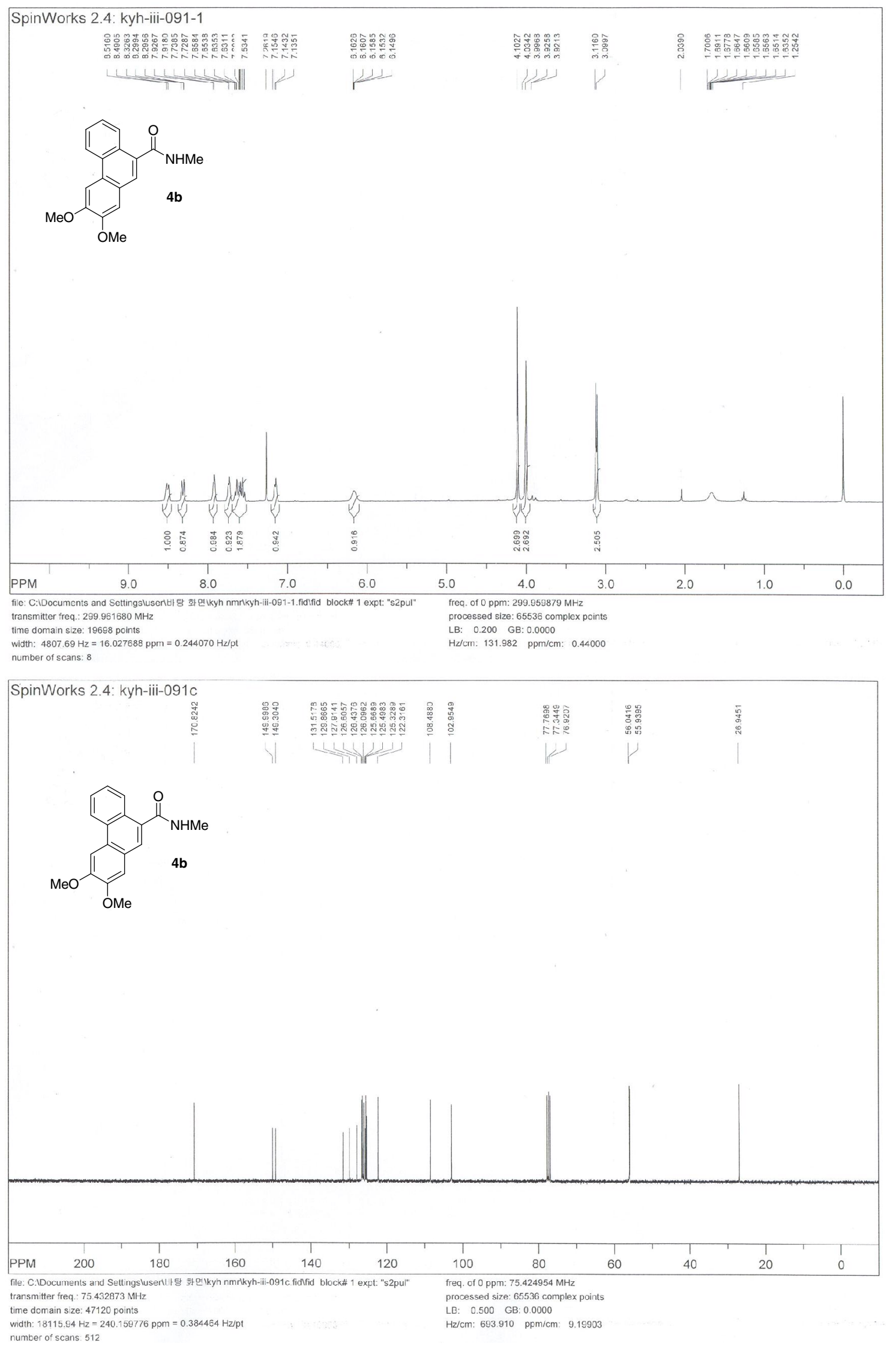




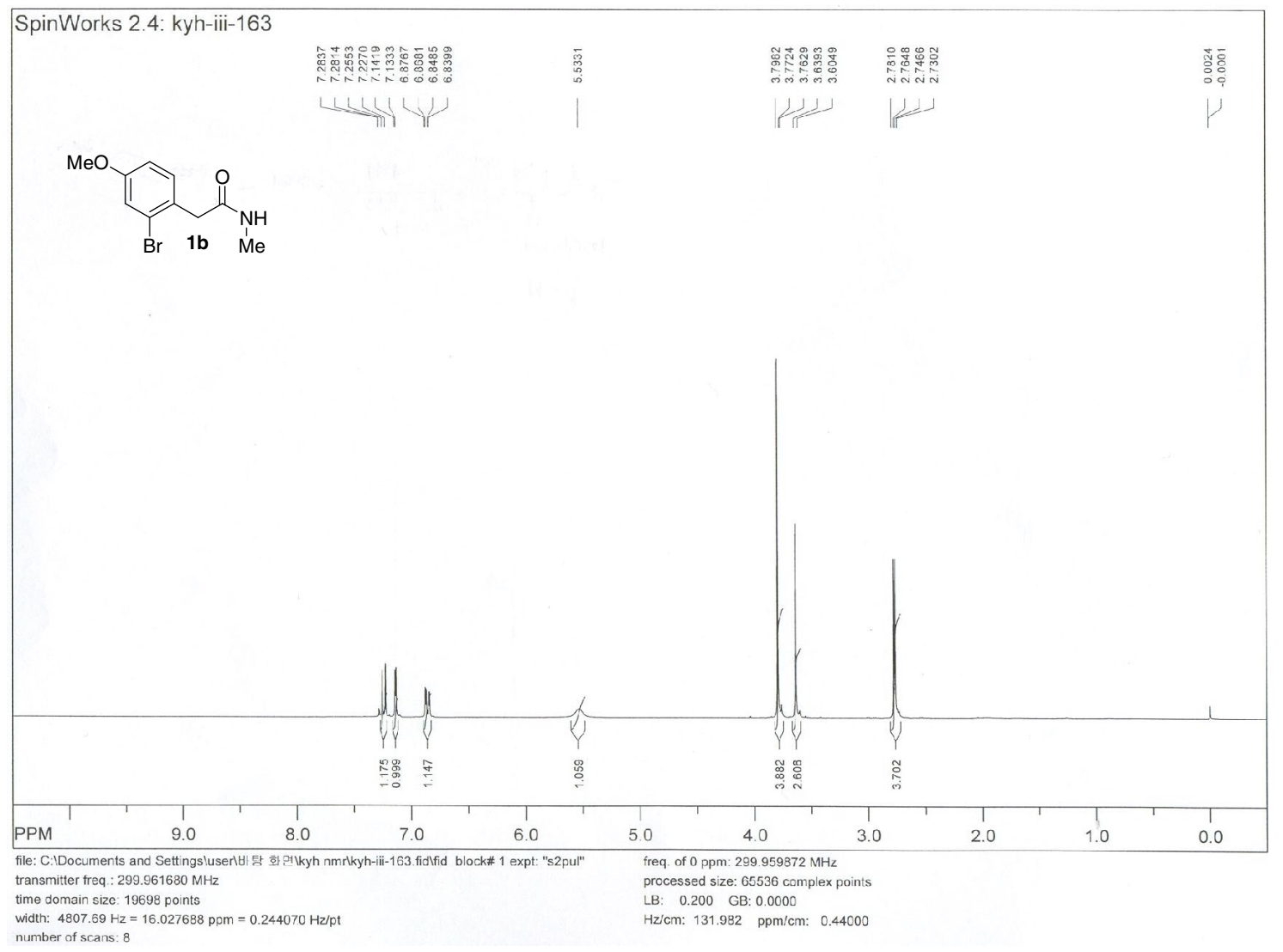

\begin{tabular}{|c|c|c|c|c|c|}
\hline \multicolumn{6}{|l|}{ SpinWorks 2.4: kyh-iii-163c } \\
\hline & 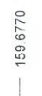 & 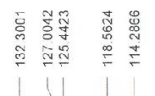 & 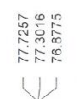 & 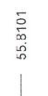 & 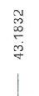 \\
\hline
\end{tabular}

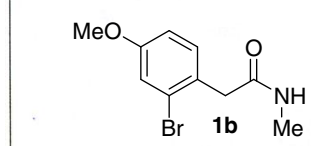




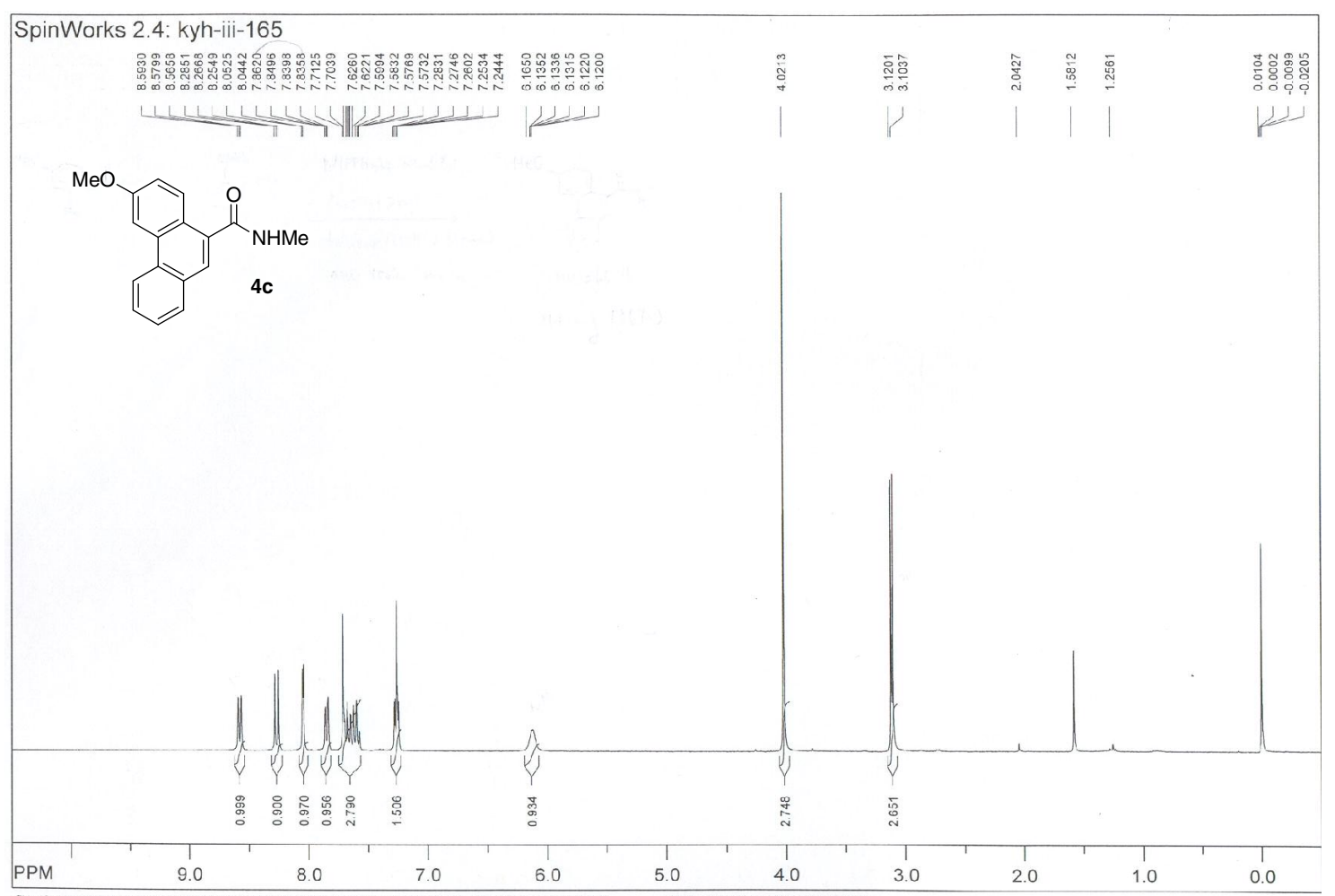

file: C:Documents and Settingsiuserl바탕 화연/kyh nmrlkyh-iii-165.fidffid block\#1 1 expt: "s2pul" freq. of O ppm: $299.959879 \mathrm{MHz}$

transmitter freq.: $299.961680 \mathrm{MHz}$

time domain size: 19698 points

processed size: 65536 complex points

width: $4807.69 \mathrm{~Hz}=16.027688 \mathrm{ppm}=0.244070 \mathrm{~Hz} / \mathrm{pl}$

LB: 0.200 GB: 0.0000

number of scans: 8

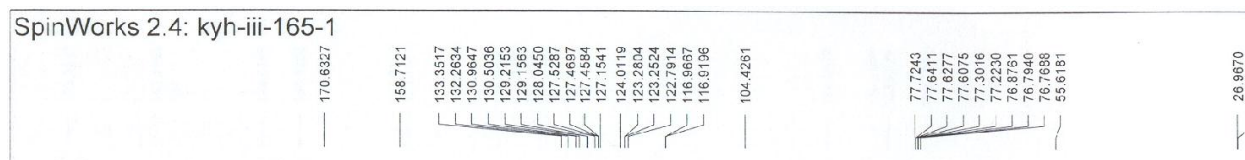

$\mathrm{MeO}$

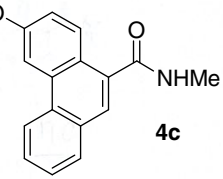

$4 c$

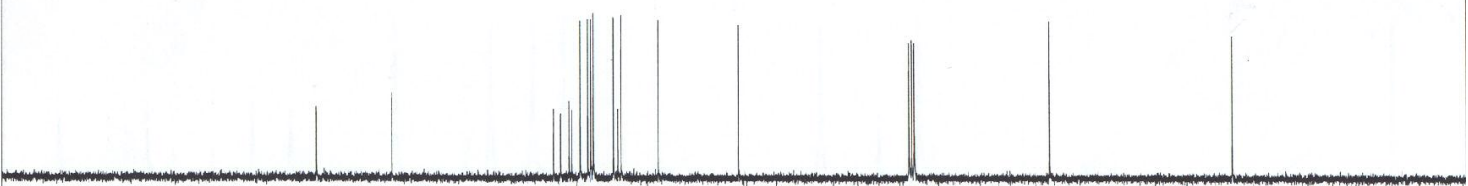

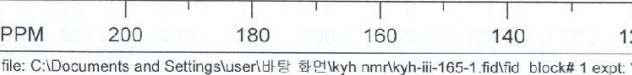

file: C:Documents and Settings

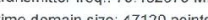

width: $18115.94 \mathrm{~Hz}=240.15$

$159776 \mathrm{ppm}=0.384464 \mathrm{~Hz} / \mathrm{pt}$

Dumber of scans: 512

$1+1$

100

80

60

40

20

freq. of O pprn: $75.424954 \mathrm{MHz}$

processed size: 65536 complex points

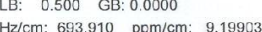

\section{商}

(n) 

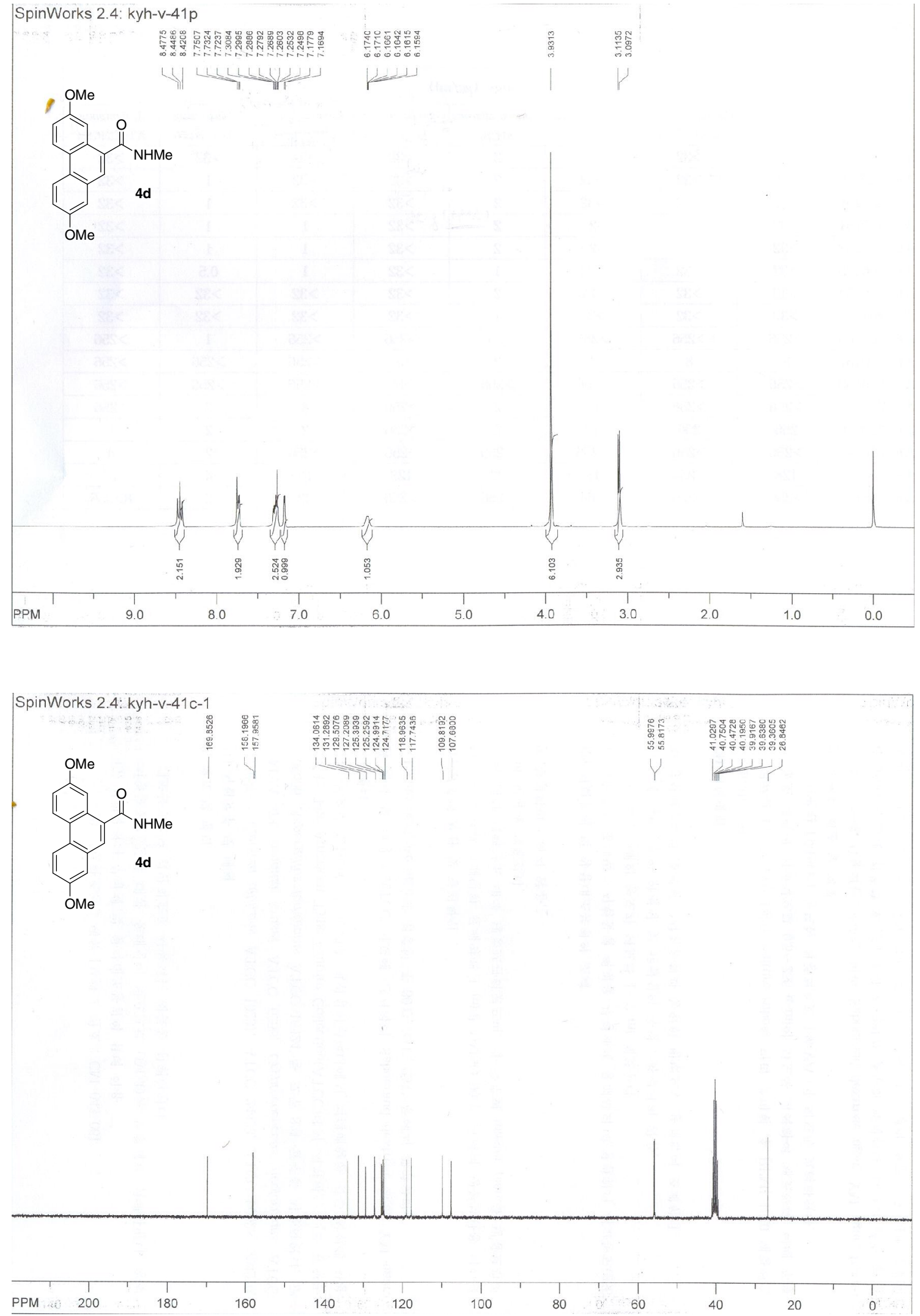


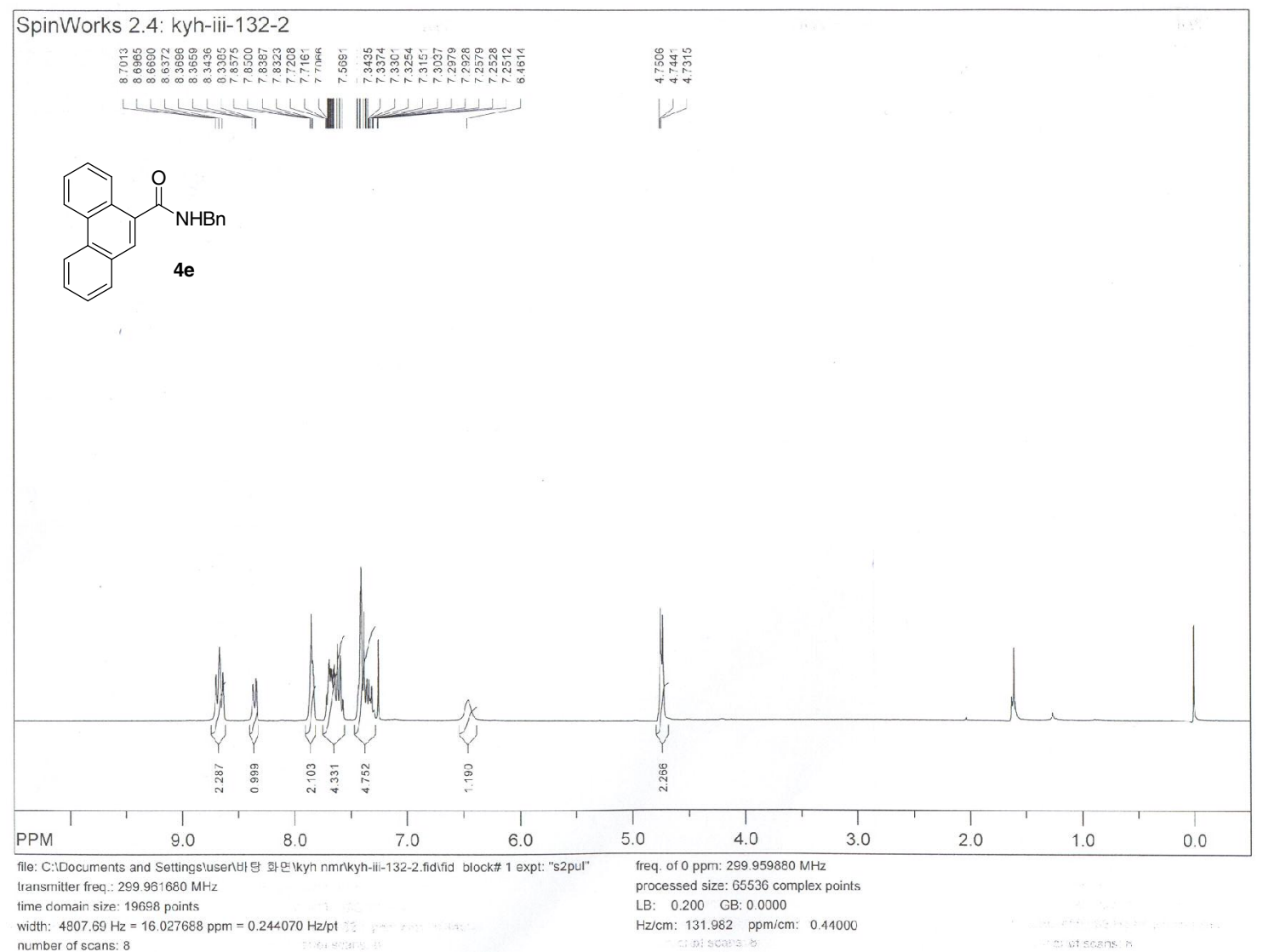
number of scans: 8

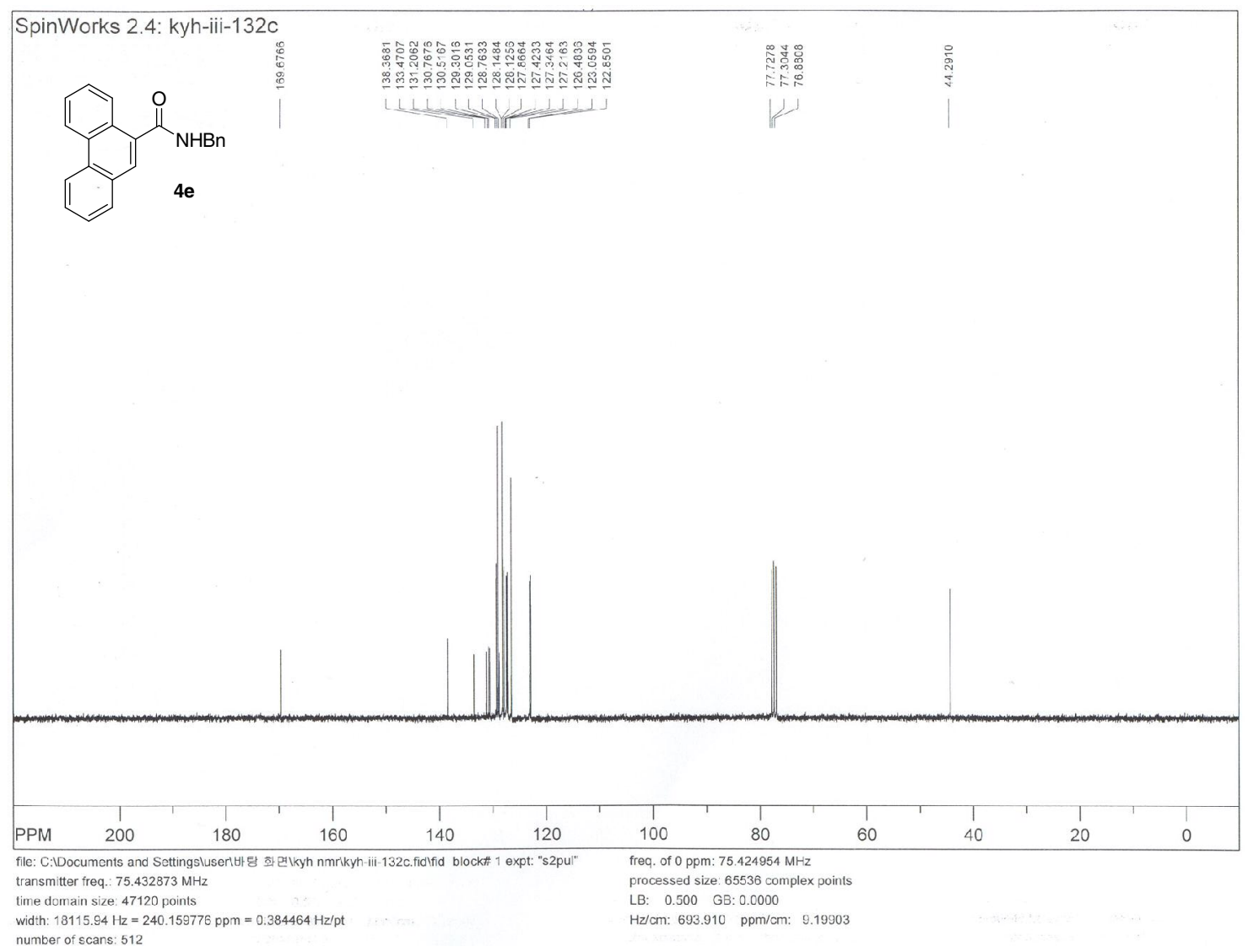



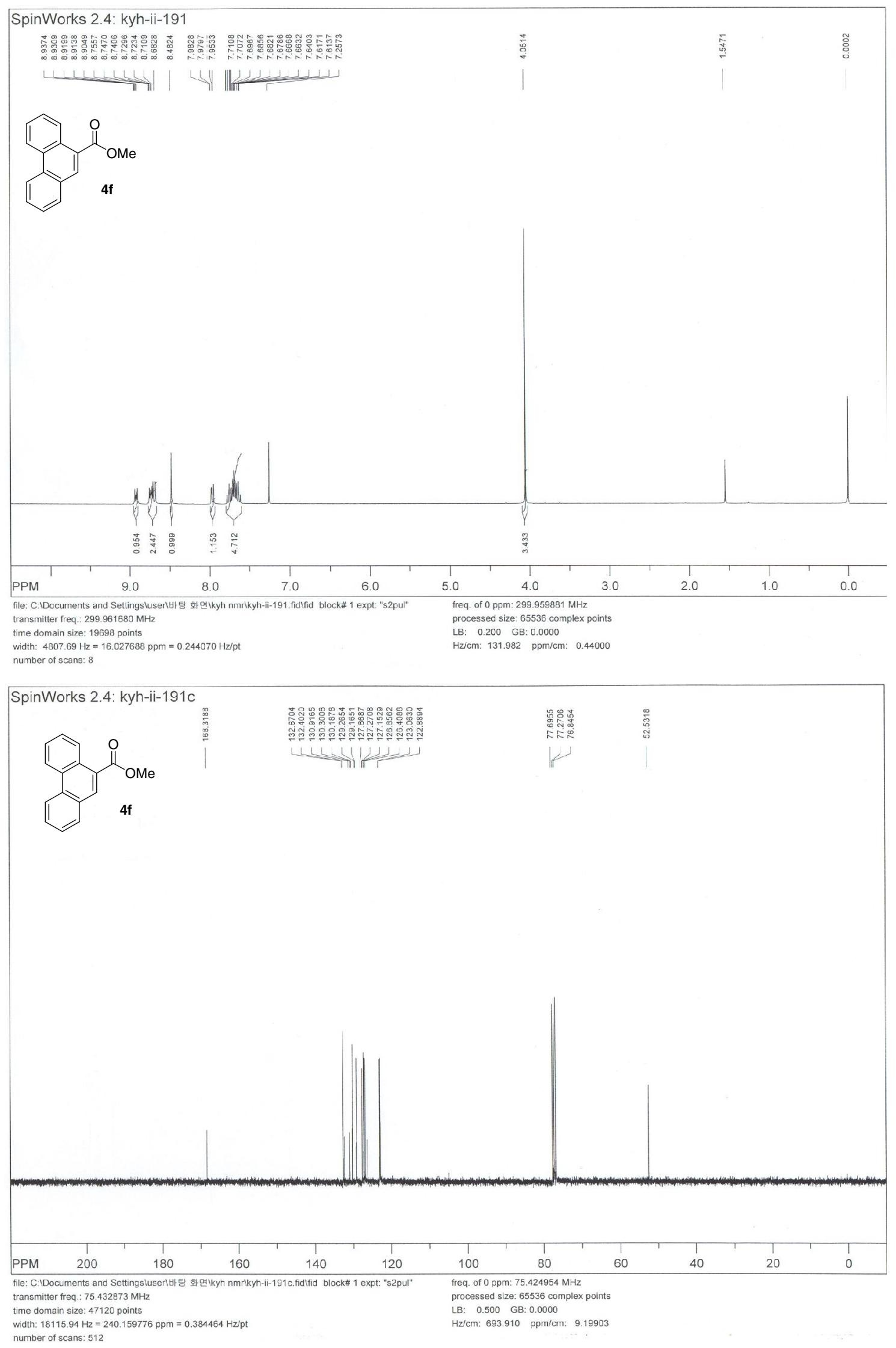

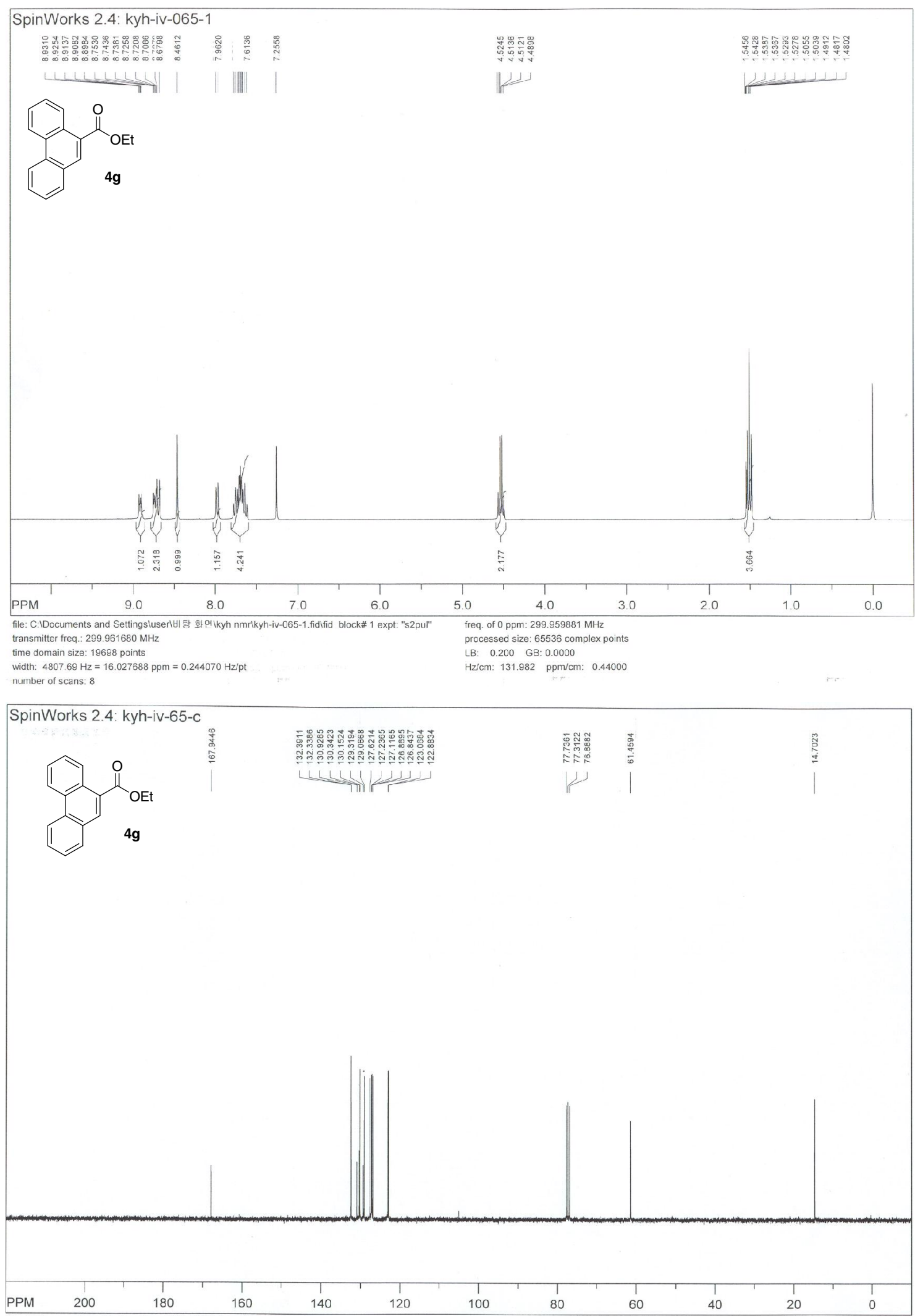

file: C:DDocuments and Settingslusent바당 회년 kyh nmrlkyh-iv-kkyh-iv-65-c. fidlfid block\# 1 expt: "s2pul" freq. of 0 ppm: $75.424954 \mathrm{MHz}$ transmitter freq: $75.432873 \mathrm{MHz} \quad$ processed size: 65536 complex points Whe do

width: $18115.94 \mathrm{~Hz}=240.159776 \mathrm{ppm}=0.384464 \mathrm{~Hz} / \mathrm{pt}$. ppm/cm. 


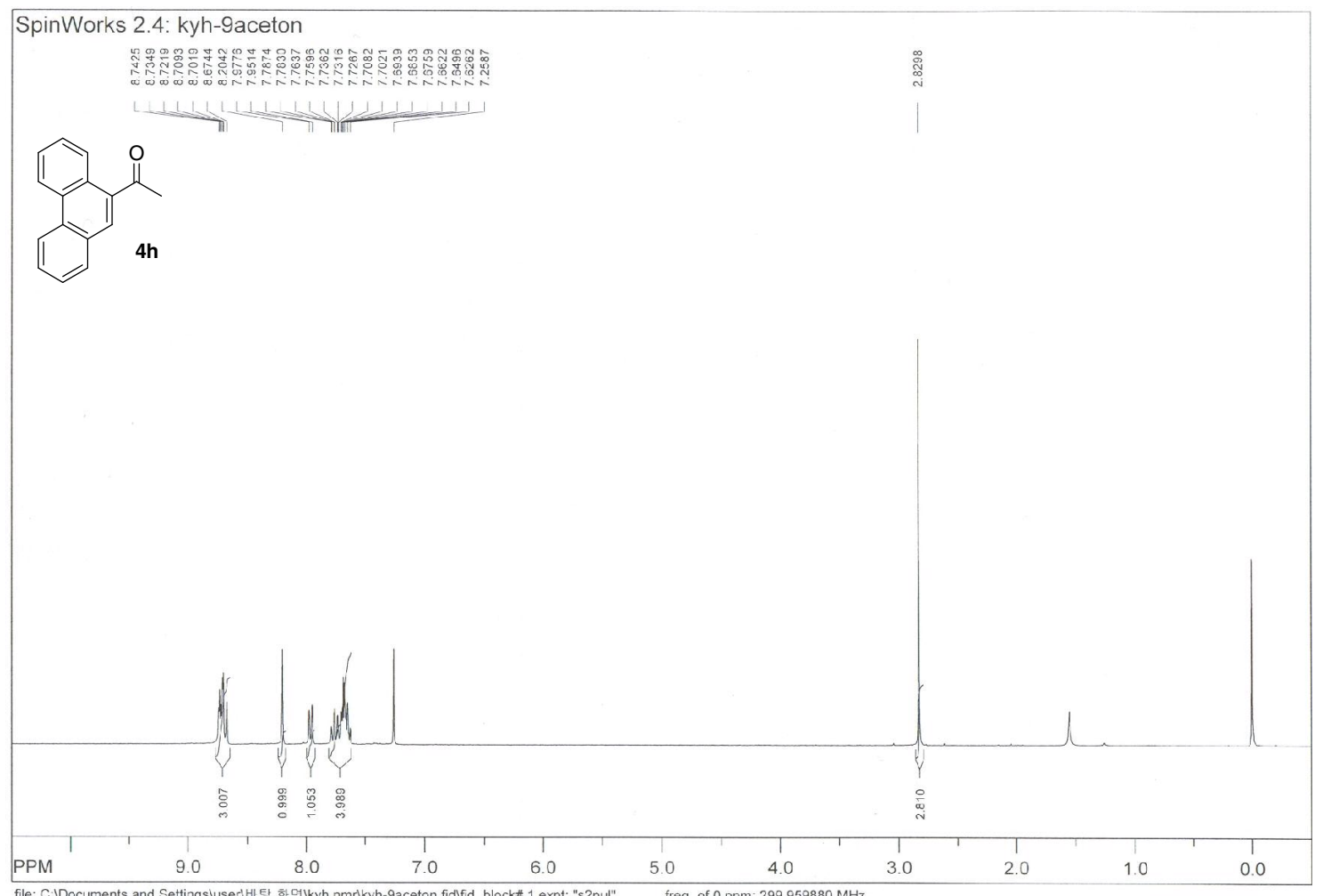

file: C:IDocuments and Settingsiuseri바탕 화던(kyh nmrkkyh-9aceton. fidlfid block\# 1 expt: "s2pul" transmitter freq.: $299.961680 \mathrm{MHz}$

time domain size: 19698 points
width: $4807.69 \mathrm{~Hz}=16.027688 \mathrm{ppm}=0.244070 \mathrm{~Hz} / \mathrm{pt}$

freq. of o ppm: $299.959880 \mathrm{MHz}_{2}$

processed size: 65536 comp
LB: $0.200 \quad$ GB: 0.0000

number of scans:

$\mathrm{Hz} \mathrm{z}^{\prime} \mathrm{cm}: 131.982 \mathrm{ppm} / \mathrm{cm}: 0.44000$
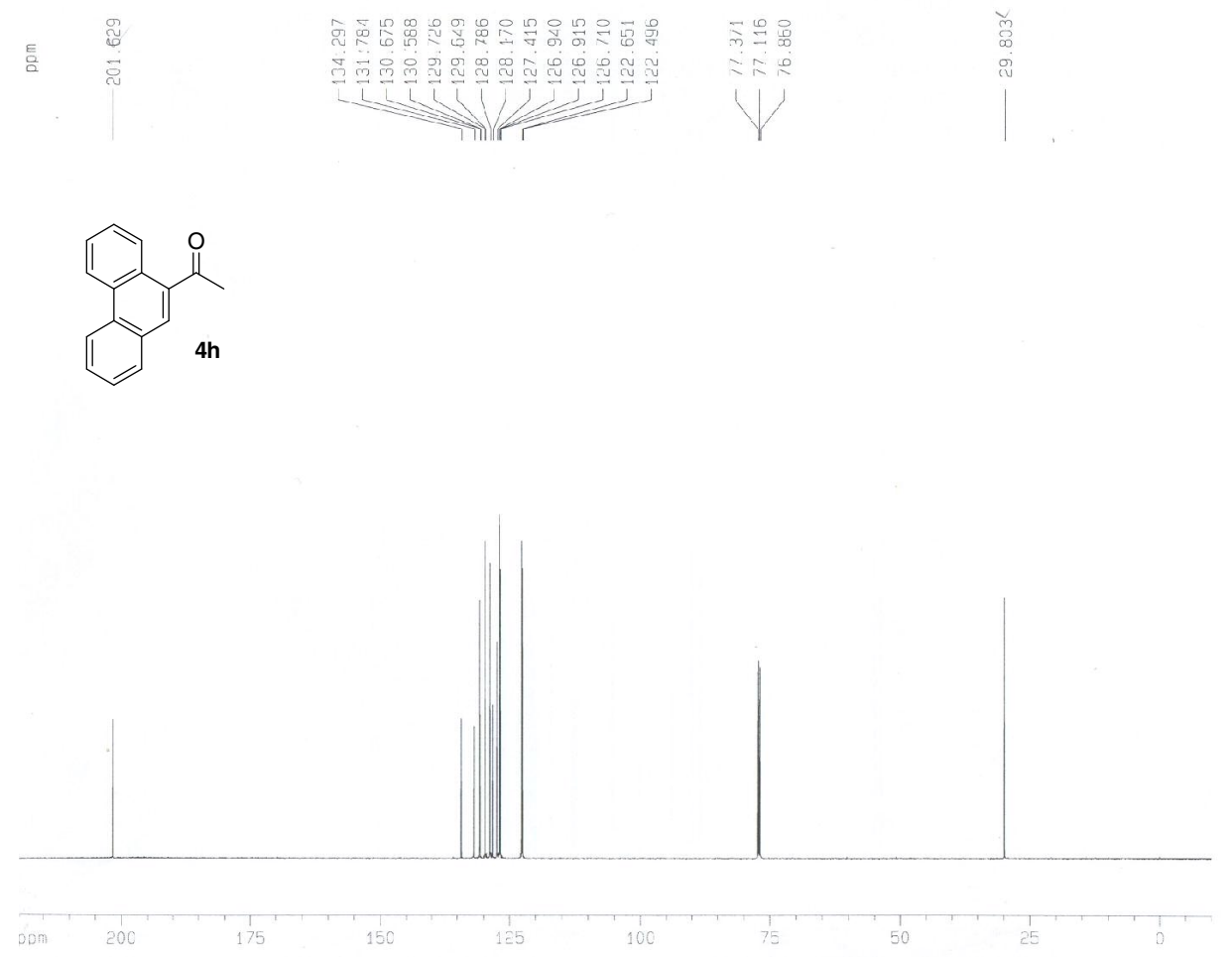

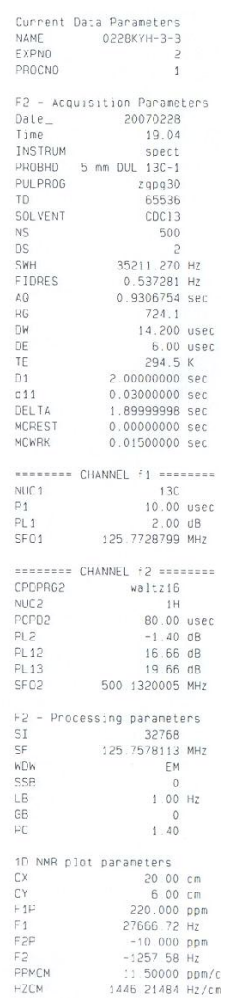




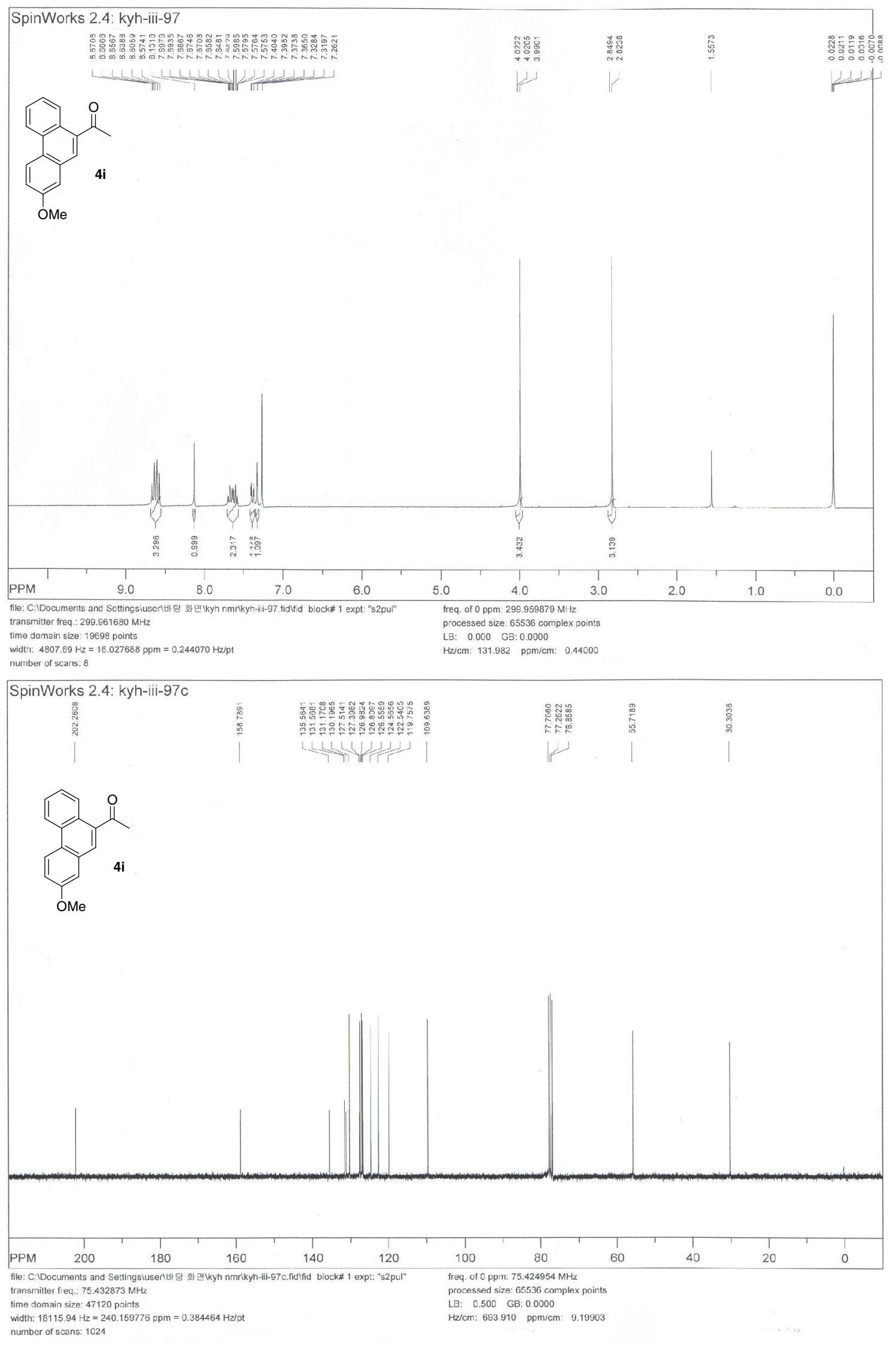



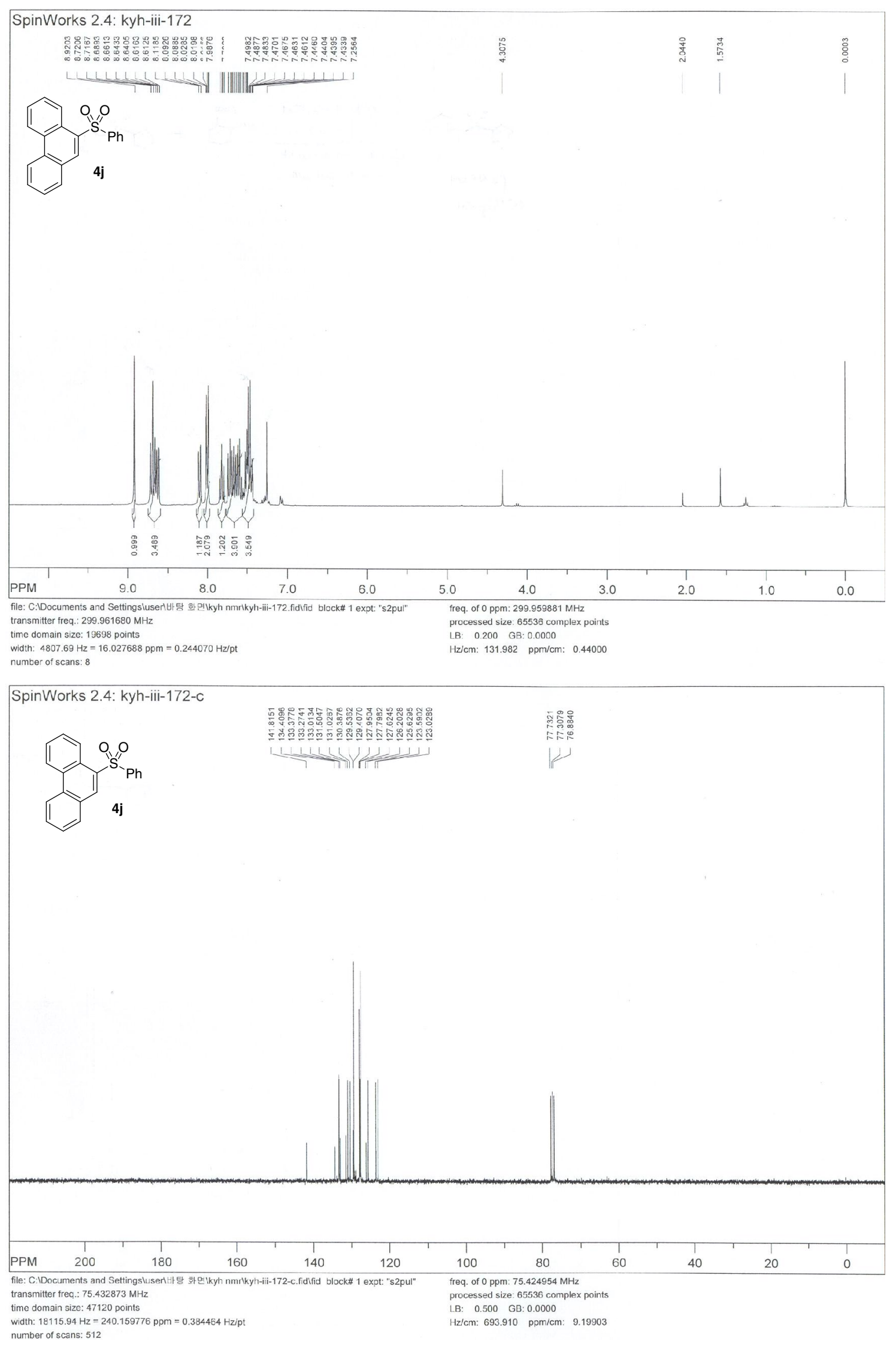


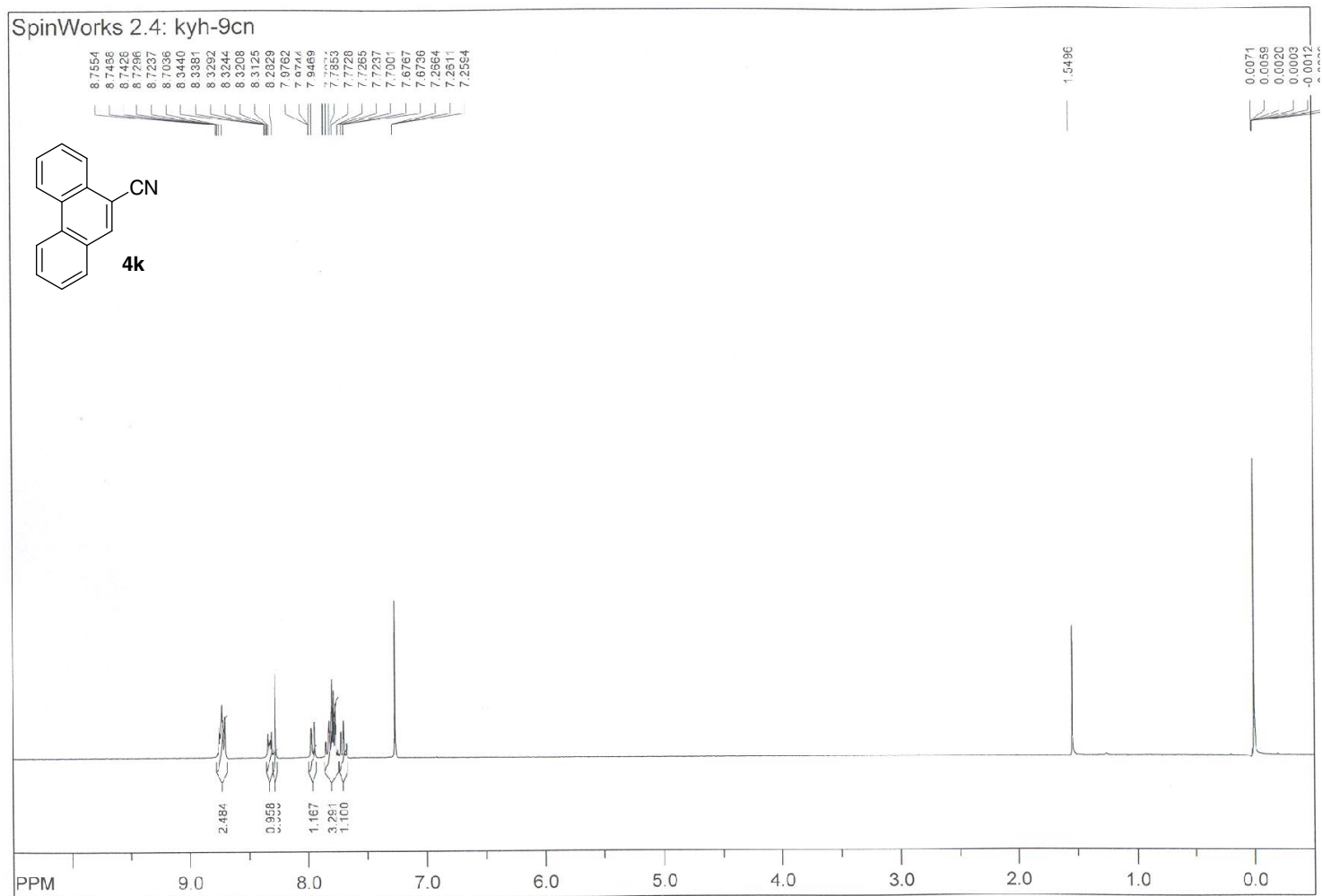

file: C.:Documents and Settingsiuserl바탐 화여kyh nmrikyh-9cn.fidffid block\# 1 expt: "s2pul" $\quad$ treq. of o ppm: $299.959878 \mathrm{MHz}$

transmitter freq: $299.961680 \mathrm{MHz} \quad$ processed size: 65536 complex points $\begin{array}{ll}\text { time domain size: } 19698 \text { points } & \text { LB: } 0.200 \text { GB: } 0.0000\end{array}$

vidith: $4807.69 \mathrm{~Hz}=16.027688 \mathrm{ppm}=0.244070 \mathrm{~Hz} / \mathrm{pt} \quad \mathrm{Hz} / \mathrm{cm}: 131.982 \mathrm{ppm} / \mathrm{cm}: 0.44000$

not

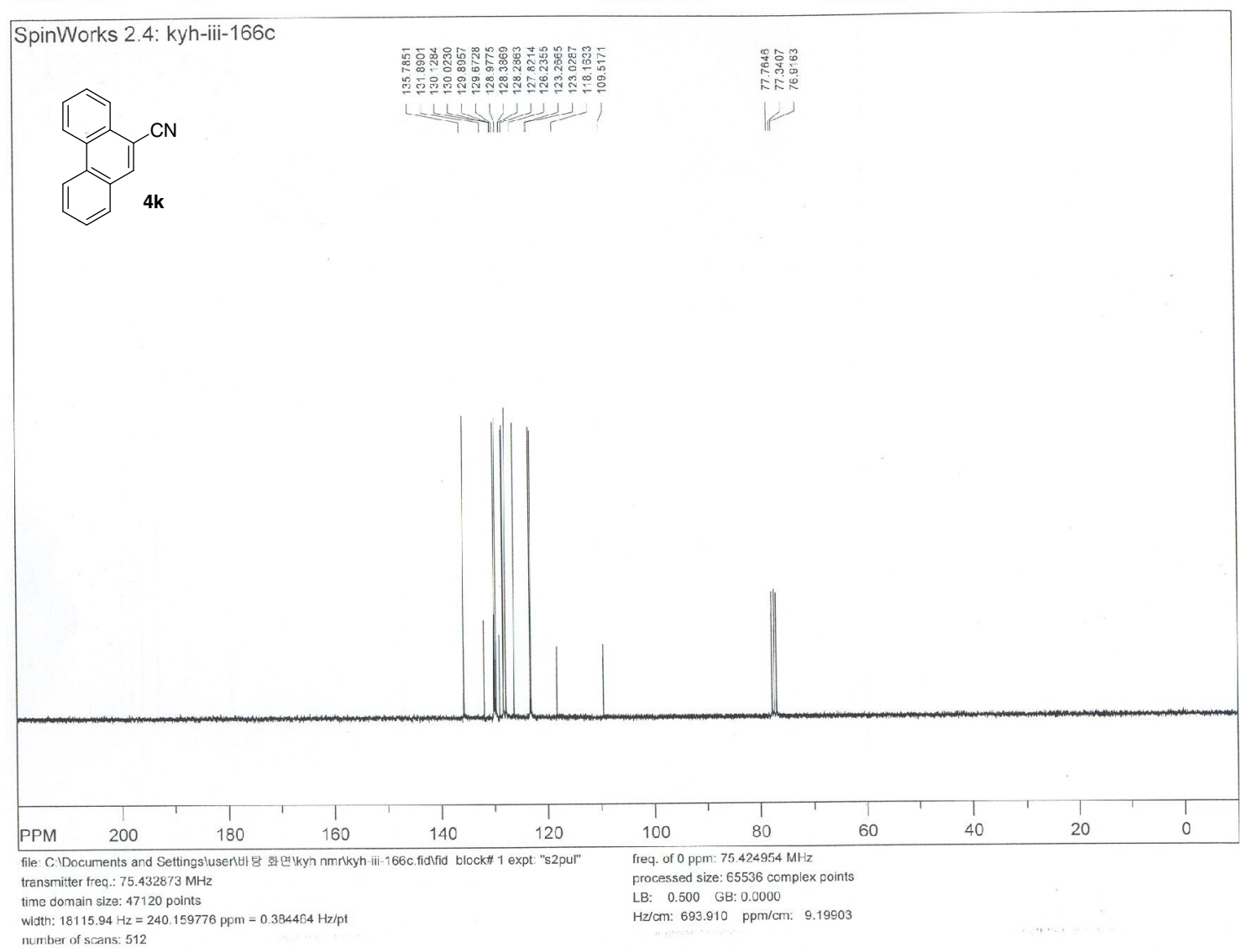




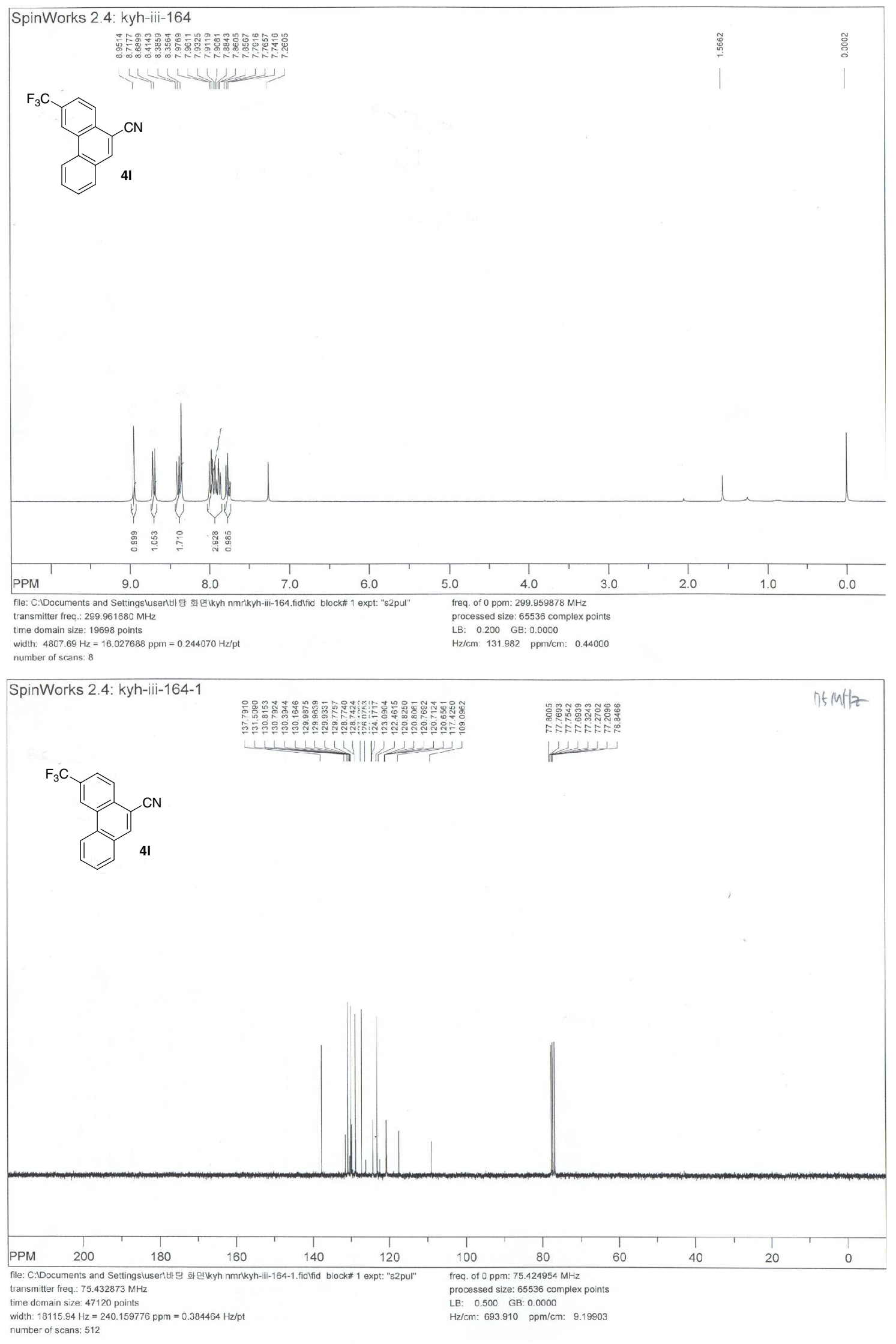




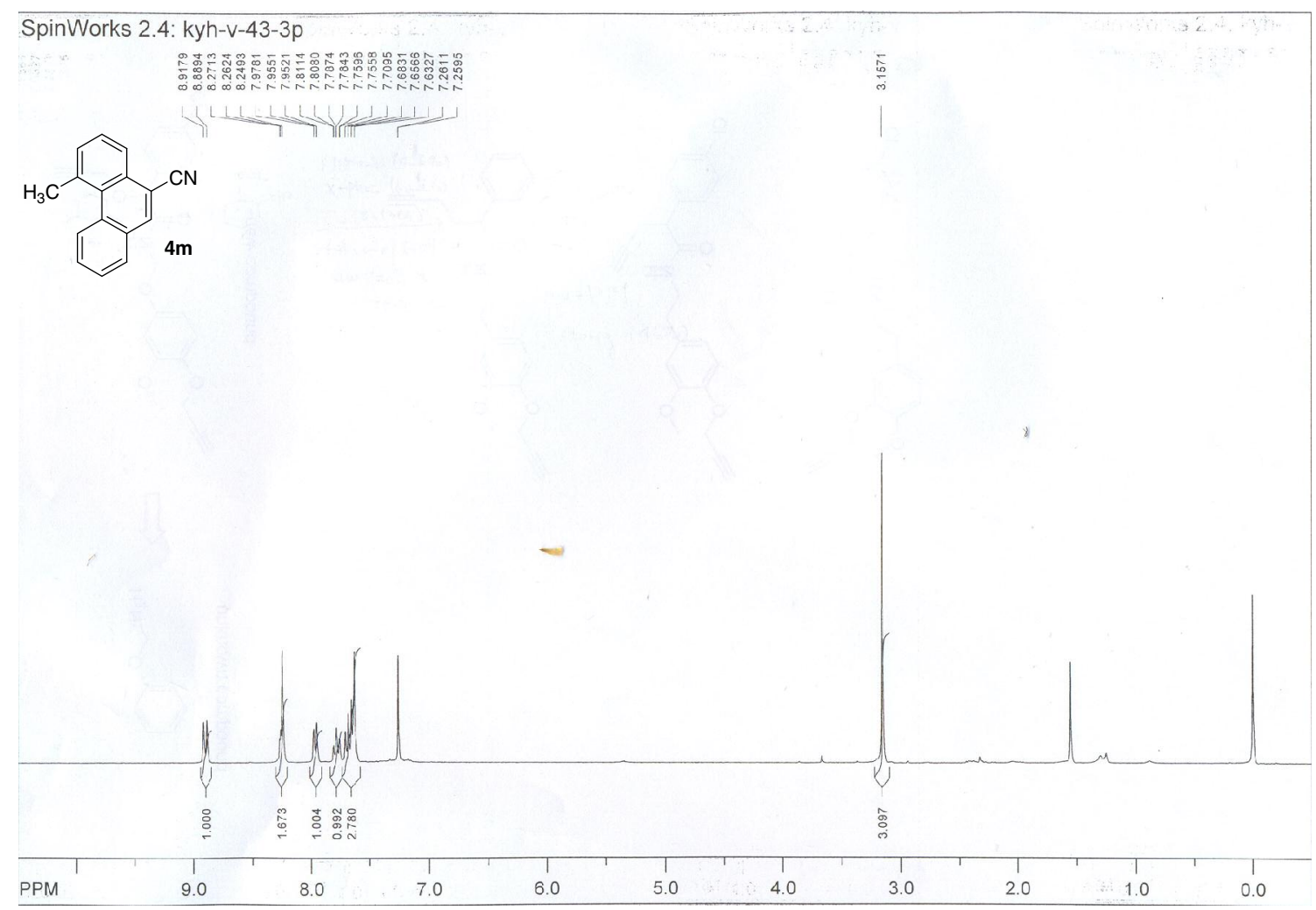

SpinWorks 2.4: kyh-v-43-3c<smiles>Cc1cccc2c(C#N)cc3ccccc3c12</smiles>
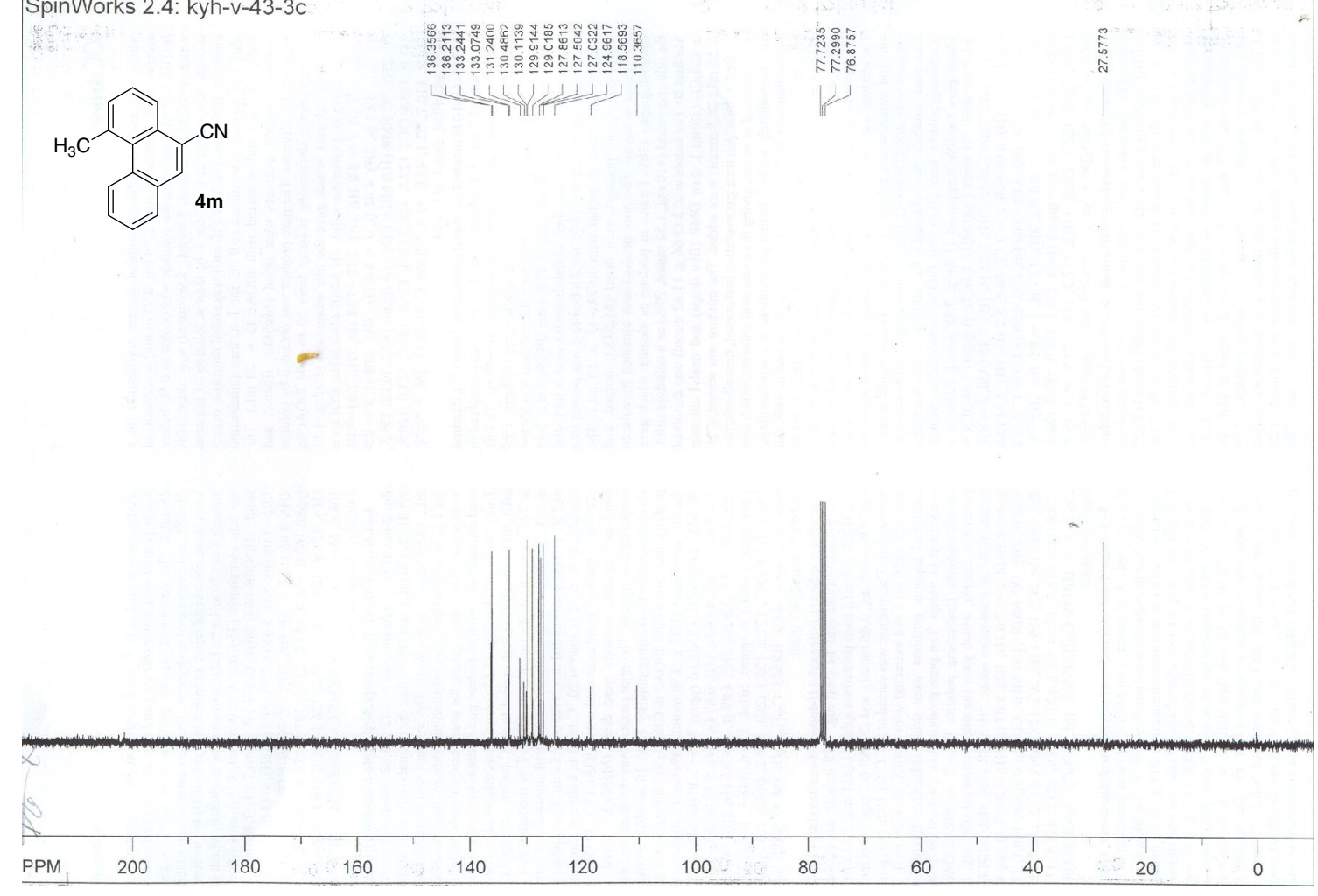

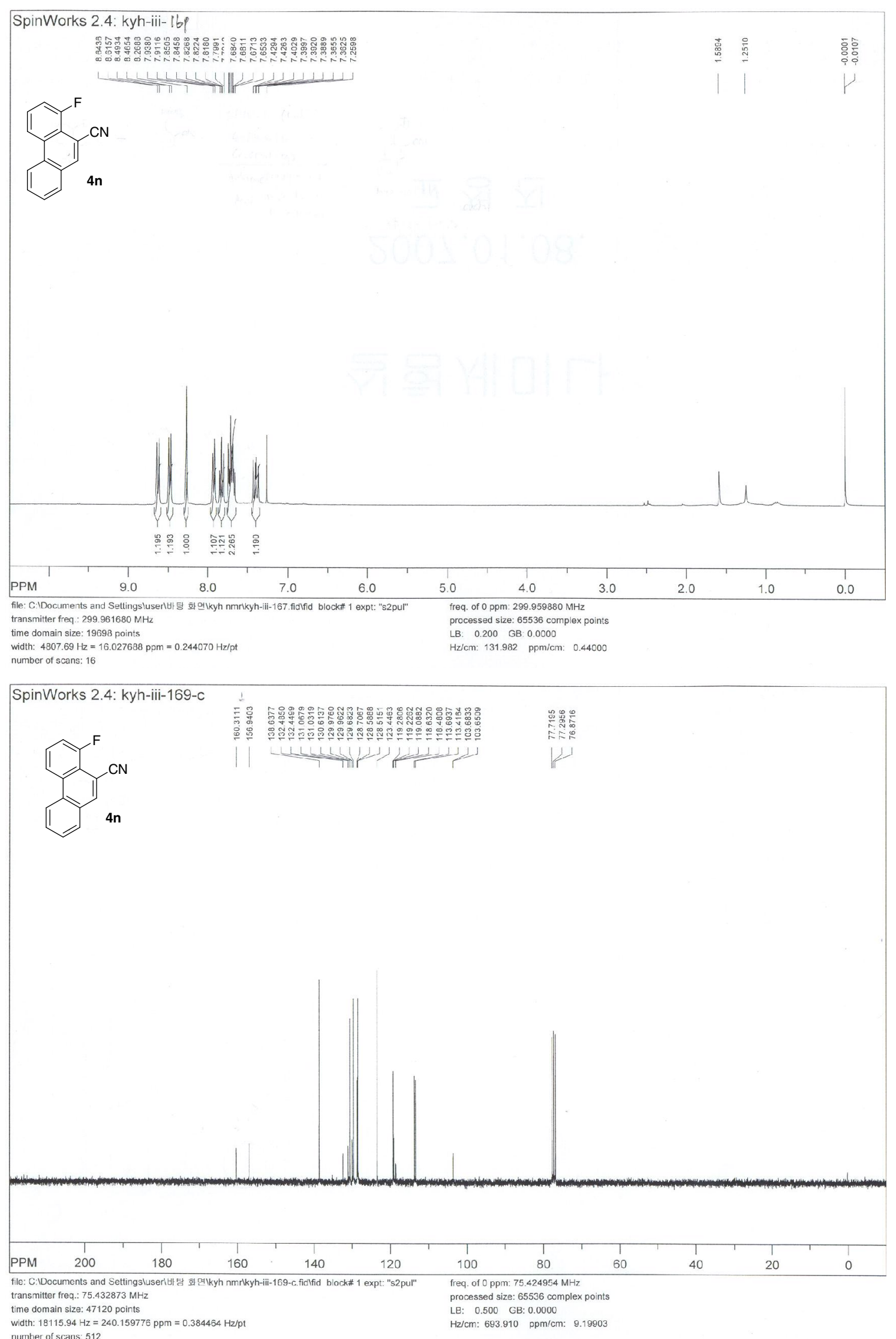


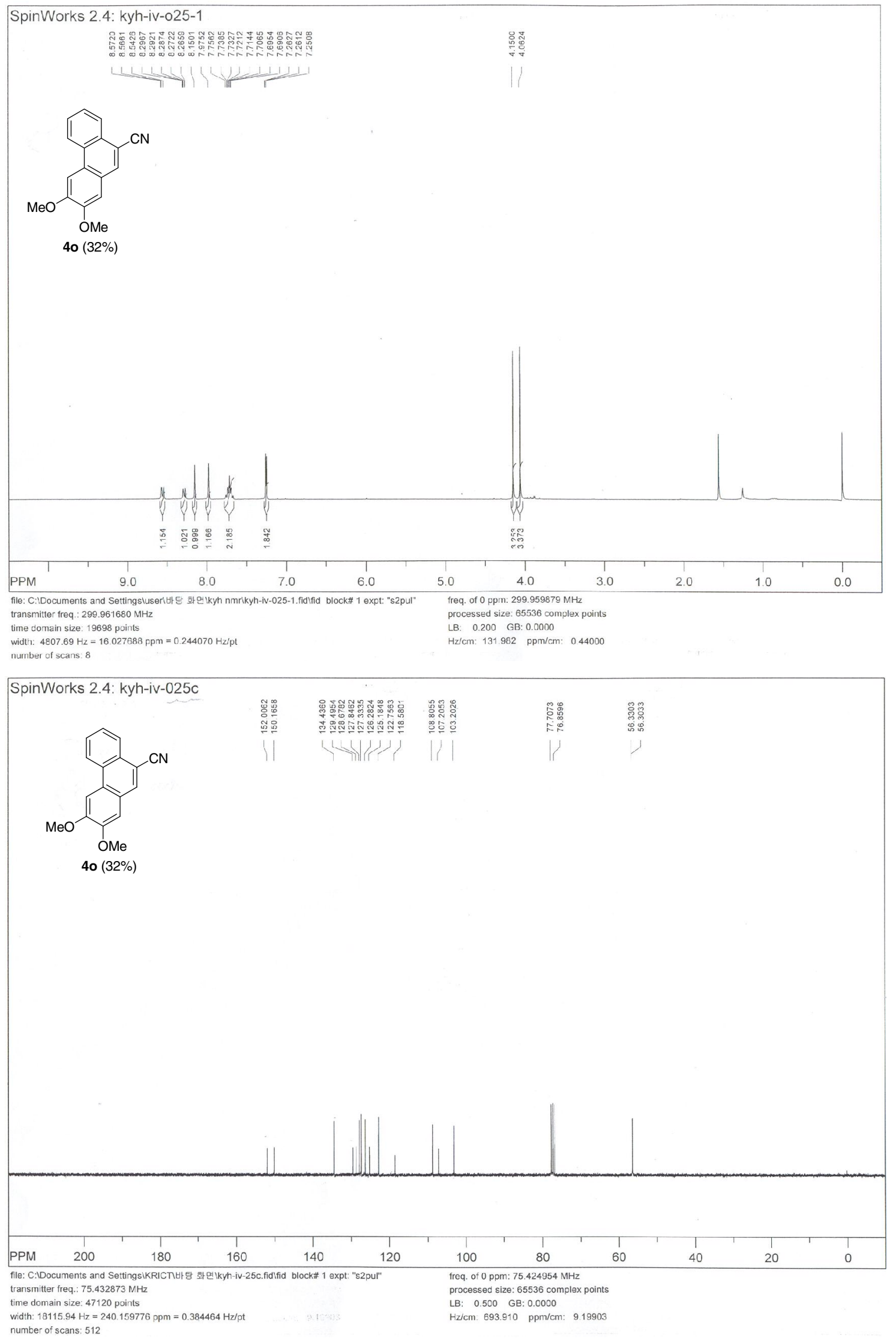



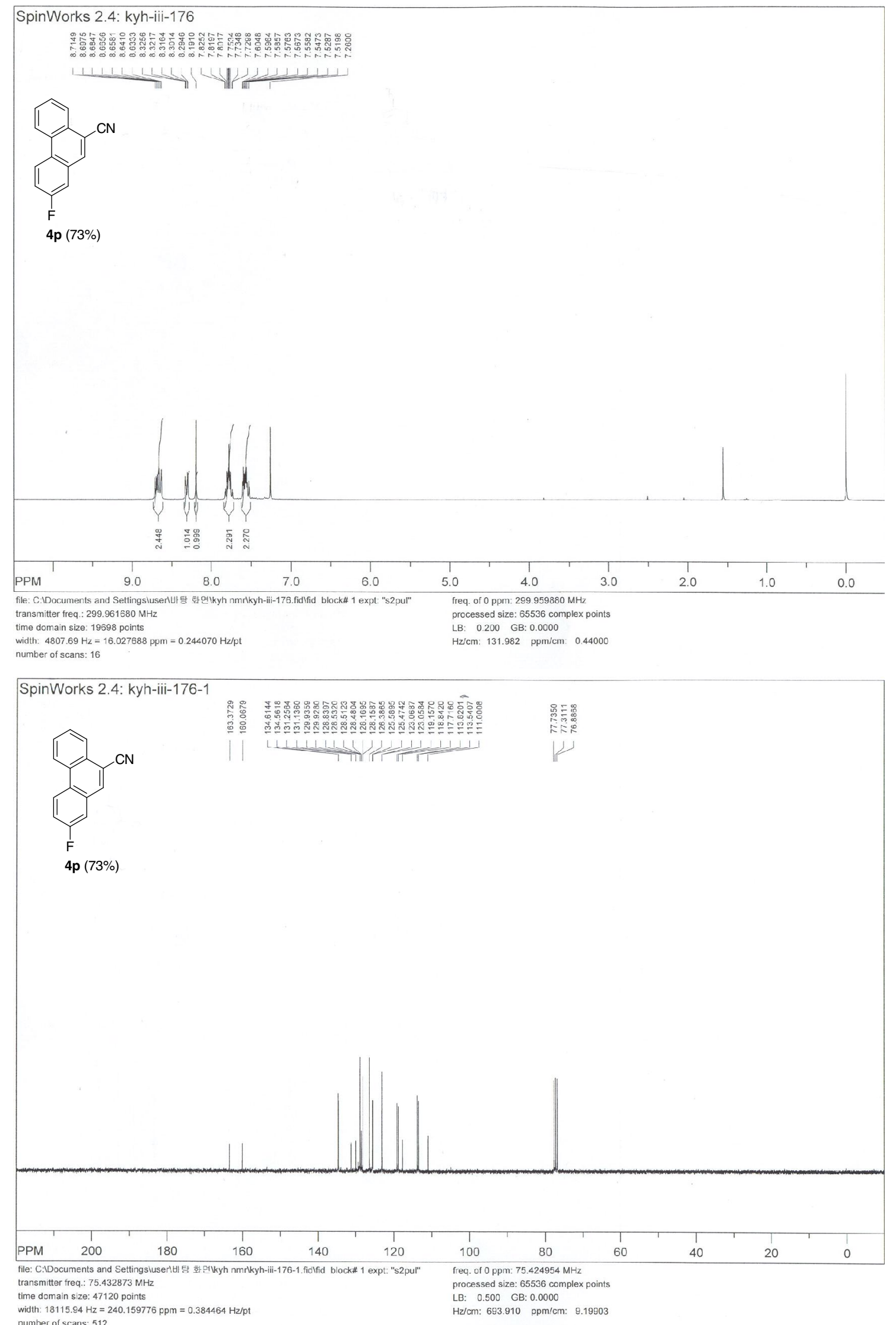


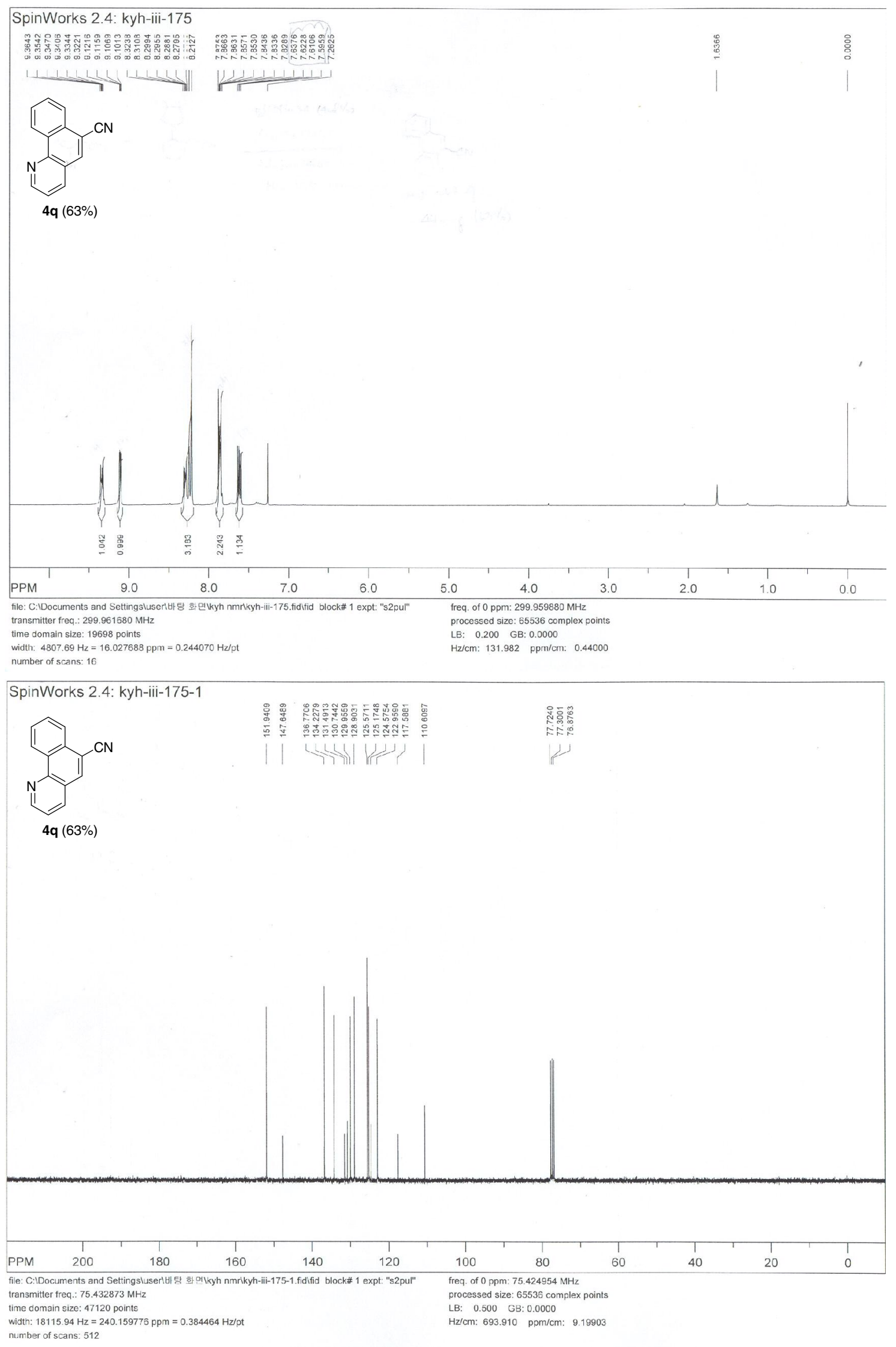



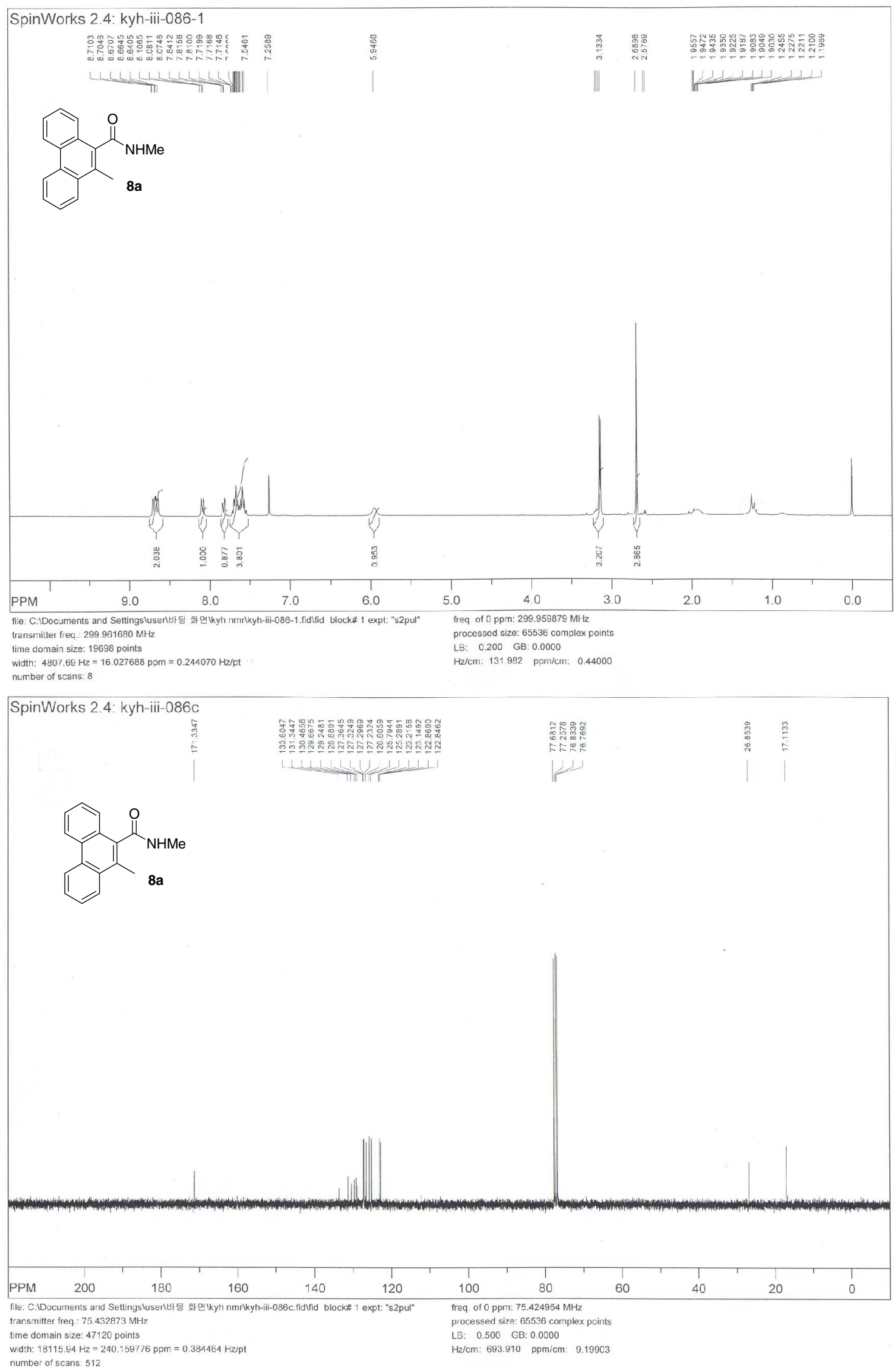

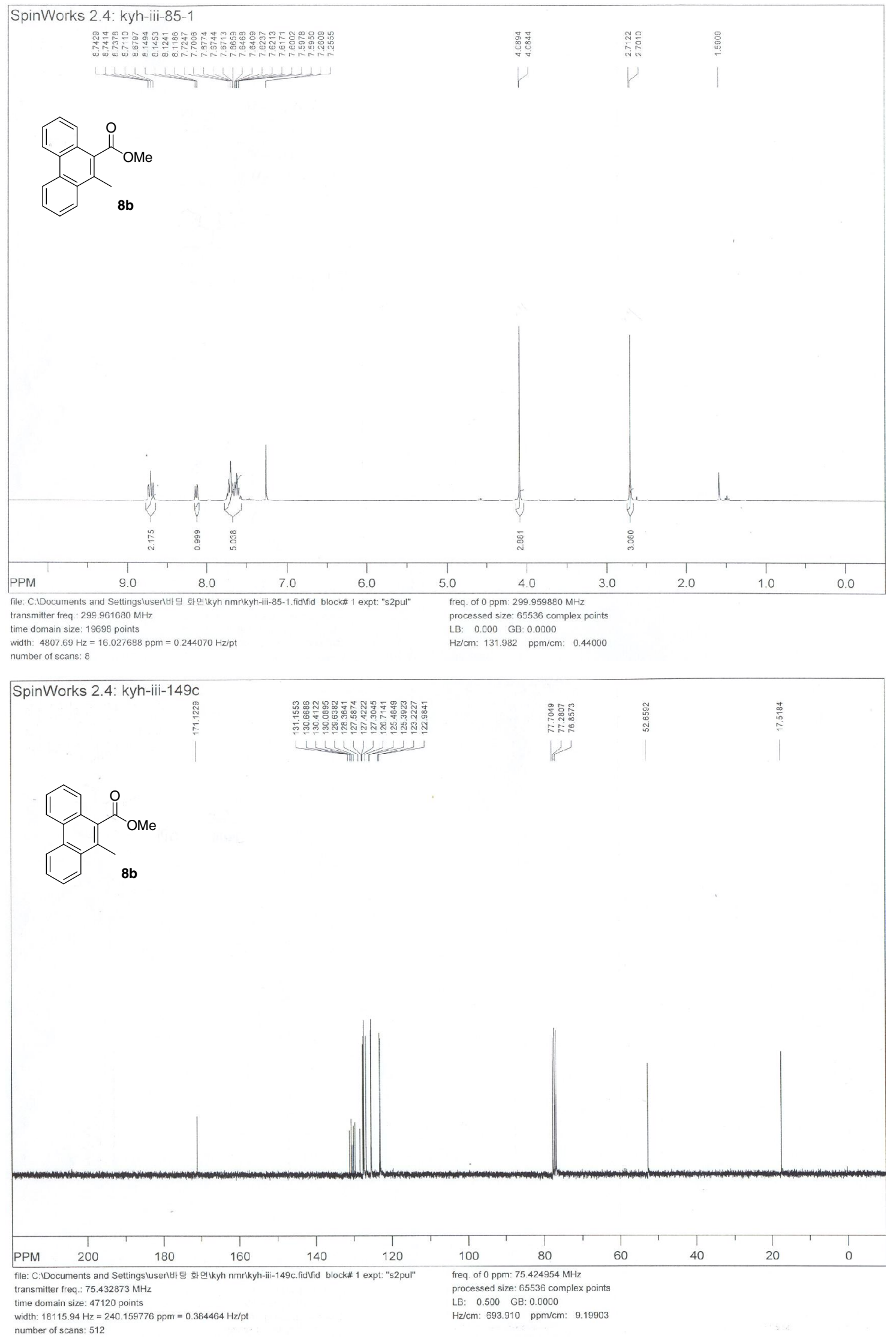


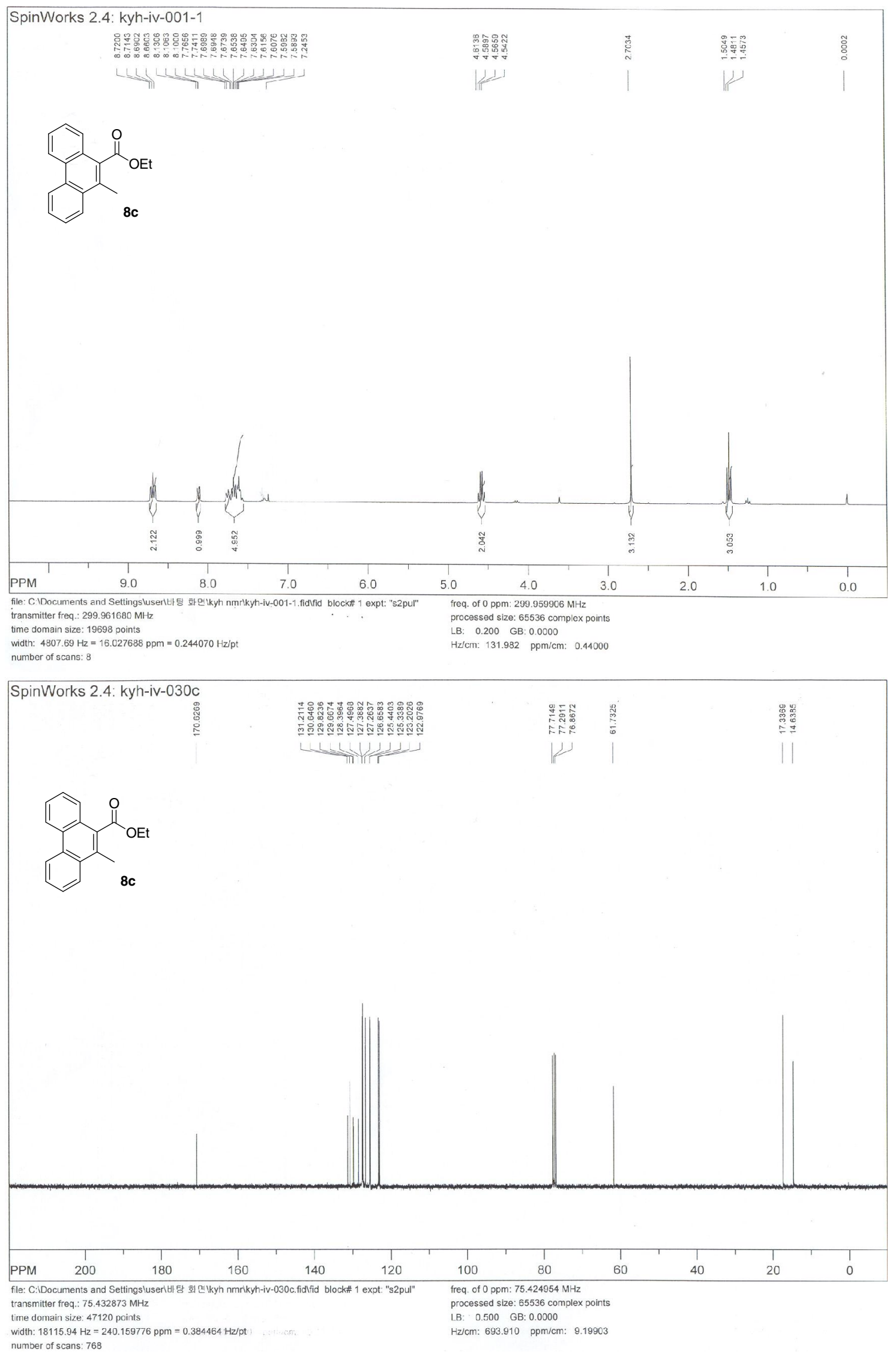




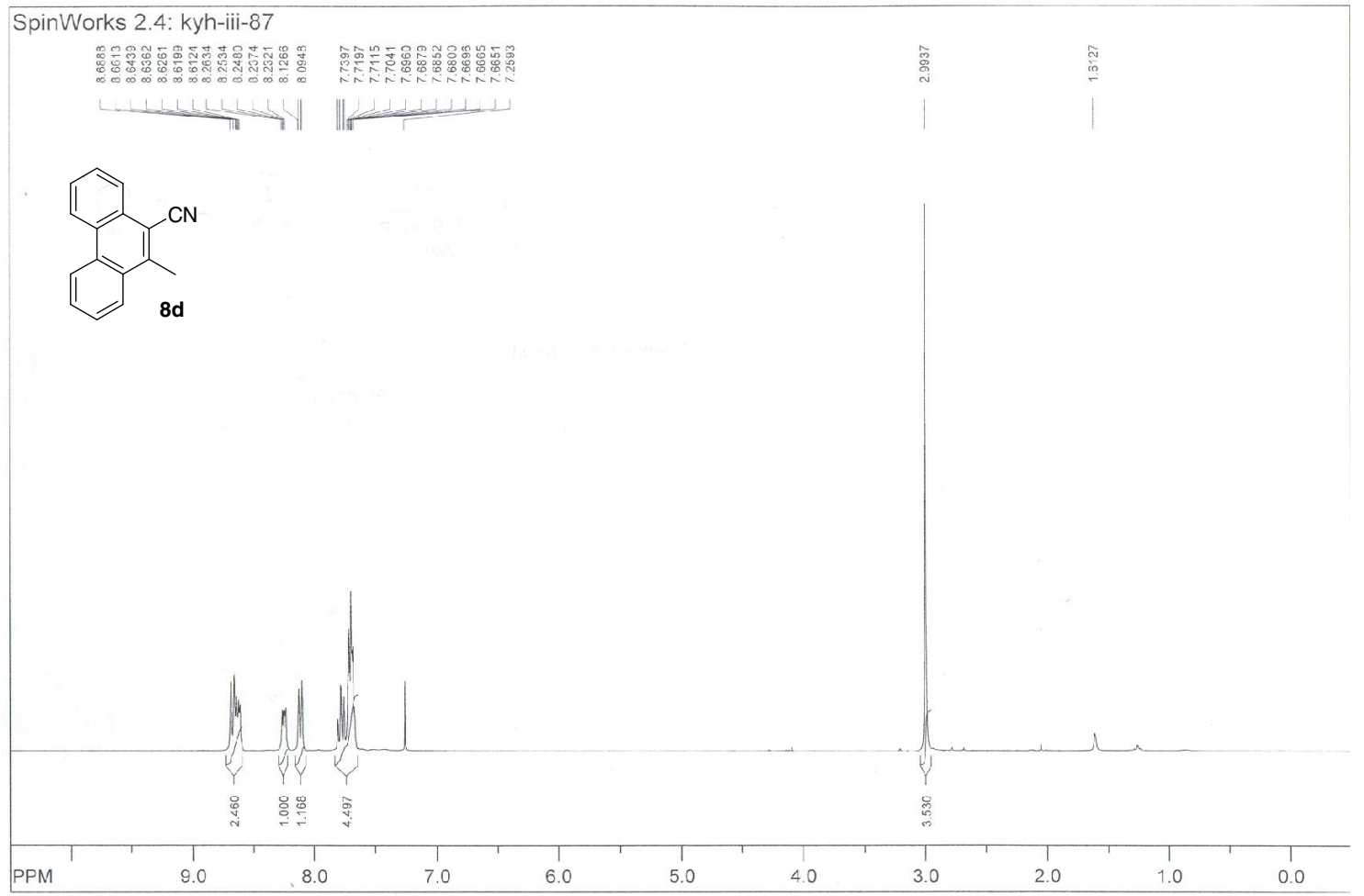

file: C:Documents and Settingslusert바탕 화련|kyh nmrikyh-ii-87. fidffid block\# 1 expt: "s2pul" $\quad$ freq. of 0 ppm: $299.959880 \mathrm{MHz}$

$\begin{array}{ll}\text { transmitter freq.: } 299.961680 \mathrm{MHz} & \text { processed size: } 65536 \text { complex points } \\ \text { time domain size: } 19698 \text { points } & \text { LB: } 0.200 \text { GB: } 0.0000\end{array}$

width: $4807.69 \mathrm{~Hz}=16.027688 \mathrm{ppm}=0.244070 \mathrm{~Hz} / \mathrm{p}$

number of scans: 8

(9Pग) $\mathrm{K} Y \mathrm{H}-3-10$<smiles>Cc1c(C#N)c2ccccc2c2ccccc12</smiles>

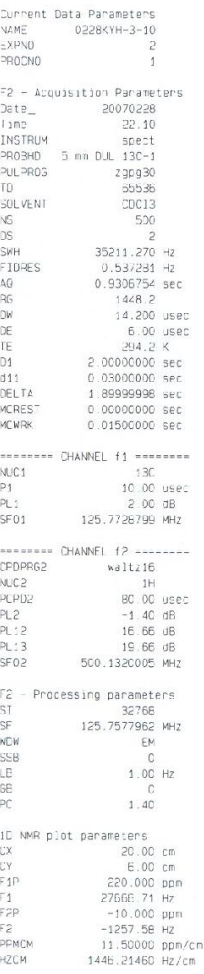



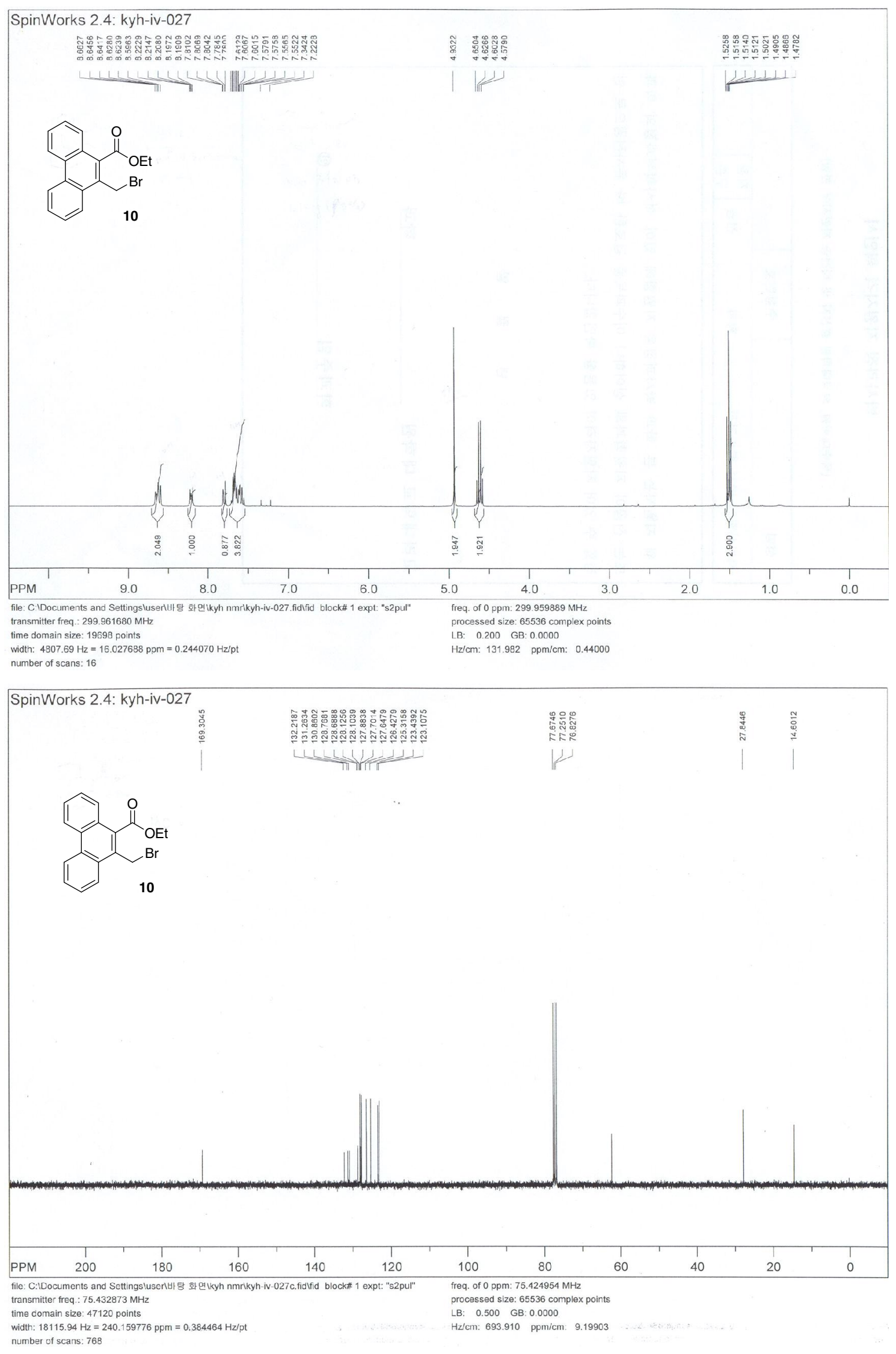

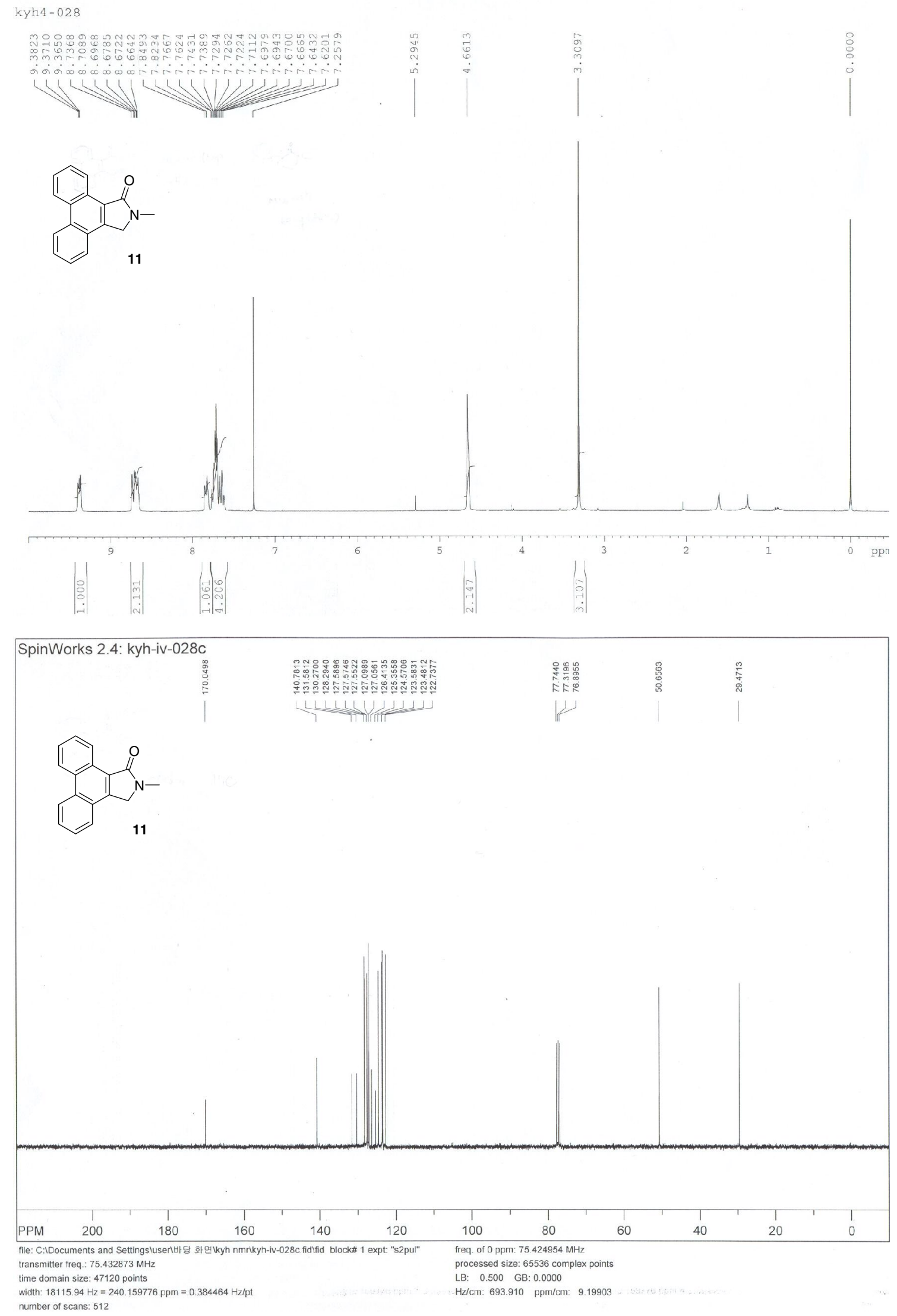